\title{
A comparative ethno-botanical study of Cholistan (an arid area) and Pothwar (a semi-arid area) of Pakistan for traditional medicines
}

\author{
Sadia Malik', Saeed Ahmad², Alia Sadiq ${ }^{1}$, Khurshid Alam³ ${ }^{3}$ Hafiz Muhammad Wariss³, Imtiaz Ahmad ${ }^{3}$, \\ Muhammad Qasim Hayat ${ }^{1 *}$, Shazia Anjum ${ }^{3^{*}}$ and Muhammad Mukhtar ${ }^{4}$
}

\begin{abstract}
Background: The present study is intended to compare and document the therapeutic flora, their remedial use, and the traditional knowledge used frequently by the residents of the Cholistan desert and Pothwar (Potohar) Plateau of Punjab, Pakistan. The old endemic remedies of these areas are diminishing due to lack of qualitative and quantitative research.

Methods: The data was generated by unstructured-interviews, informal meetings, open-ended conversations and group discussions with local people and traditional health healers of the study area. Reported literature was also utilized.

Results: The study recorded a list of various medicinal plants used as traditional medicines by local people. Total 86 numbers of plant species belonging to 38 families and 67 plant species belonging to 29 families have been reported in the Pothwar and Cholistan respectively. Only $10.5 \%$ of similar plant species were present in the studied areas.

Conclusion: The investigation revealed that the local people of study areas inherit a rich traditional knowledge but there is great danger of losing this wealth of knowledge in the near future. Documentation of the knowledge exclusively from desert area of Cholistan, Pakistan is unique information in its nature. The study presents the undocumented knowledge worth recognition that will not only help in conservation of medicinal plant species but will highlight the pharmacological capacity for improved human healthcare regarding many common ailments.
\end{abstract}

Keywords: Medicinal plants, Traditional healthcare knowledge, Pothwar, Cholistan

\section{Introduction}

Pakistan features a diverse array of elevated peaks, snow mountains, irrigated immense plains, coasts, freezing and burning deserts. The country has been divided into four phyto-geographic regions namely the Irano-Turanian, Sino-Japanese, Saharo-Sindian and Indian element reflecting the unmatched wealth of its flora. A significant part of the country is of arid nature covering 40.9 million hectare (ha) of land together with 11 million ha of desert area. Medicinal plants play a significant role in lives of its inhabitants as they are considered as a primary source of

\footnotetext{
* Correspondence: m.qasim@asab.nust.edu.pk; anjumshazia@yahoo.com 'Medicinal Plant Research Laboratory, Department of Plant Biotechnology, Atta-ur-Rahman School of Applied Biosciences (ASAB), National University of Sciences and Technology (NUST), Islamabad, Pakistan

${ }^{3}$ Cholistan Institute of Desert Studies (CIDS), The Islamia University of

Bahawalpur, Bahawalpur, Pakistan
}

Full list of author information is available at the end of the article treatment against many diseases. They also serve as a key income resource for poor field workers and people associated with herbal products manufacturers. Majority species of medicinal plants (about 70\%) are uni-regional and rests of the species are bi-regional or pluri-regional [1]. The traditional healthcare knowledge has been passed on generally verbally over generations rather than as a written document due to which the knowledge is gradually diminishing. Additionally, increase in urbanization, growing population, habitat loss, improper documentation, over exploitation of some plant species, lack of implementation of laws and insufficient awareness are the factors contributing to the loss of this heritage [2].

Many workers have investigated the economic, ethnobotanic and medicinal importance of plants but ample research is still required [2-4]. However, during the past decade research work has been carried out in a range of 
institutions to establish the antimicrobial, anticancer, antioxidant, anti-inflammatory effects of medicinal plants [5-9]. The floristic and ethno-botanic inventories also have been made $[4,10,11]$. The patients mostly use allopathic, alternative and traditional medicines side by side without prescription of registered practitioners. Progress in development of an infrastructure and human resource to utilize medicinal plant wealth in a proper way is limited [4]. The ethno-botanic practices are common and have direct socioeconomic impacts [12]. All these facets are endured during this study. Study of the less explored desert region of the province also seems vital. In current study we have adopted partially the data from our previous studies $[3,11]$.

Data regarding ethnobotanical or ethnopharmacologically characteristics of the plants of Cholistan desert and Pothwar is almost non-existent except very few reports. The main objective of present study is to explore the relationship between local culture of folk people and plants in the pursuit of drug development and medical breakthroughs. The herbal treatments in respective regions are favored over the allopathic ones for their low cost and less side effects. The most important objective of this study is the preservation of local plant knowledge. Loss of the indigenous knowledge is a threat to the poor rural economies based on traditional livestock farming as that in the deserts like Cholistan or semi-arid area like Pothwar. It was, therefore, deemed imperative to document the ethnobotany knowledge possessed by the people of respective areas. In addition to this, present study will be a yardstick to probe standardization and systematic exploration of traditional herbs.

\section{Study area}

The total geographical area of Punjab, a province of Pakistan is 20.63 million hectares with a composition of 13.37 and 7.26 million hectares as irrigated and rain fed respectively. The two areas chosen for the present study are the Pothwar (Potohar) Plateau (Semi-arid) and the Cholistan desert (arid) (Figure 1).

The Cholistan is a desert covering an area of 26,000 $\mathrm{Km}^{2}$ located between $27^{\circ} 42^{\prime}$ and $29^{\circ} 45^{\prime} \mathrm{N}$ latitude and $69^{\circ} 52^{\prime}$ and $75^{\circ} 24^{\prime}$ E longitude (Figure 1) at a height of $112 \mathrm{~m}$ above sea level [13-16]. Its old civilization has vanished mainly due to a variety of hostile invading problems caused by the Egyptian, Harappan and Mesopotamian civilizations [17]. The prominent climatic features of the Cholistan desert are sub-tropical, arid, burning hot, monsoon rainfall with intermittent long droughts and strong summer winds having relatively low humidity and high rate of evaporation [18]. The mean annual rainfall varies between $100 \mathrm{~mm}$ in the West and $250 \mathrm{~mm}$ in the East, with heavy showers during July to September in monsoon and January to March during winter. The mean summer temperature is $34-38^{\circ} \mathrm{C}$, while that of winter is $15-20^{\circ} \mathrm{C}$. The June being the hottest experiences $45^{\circ} \mathrm{C}$ normally and sometimes rises as high as $51^{\circ} \mathrm{C}$ [19].

The desert is separated into two eco-regions by old Hakra River. The northern division covers about $7,770 \mathrm{~km}^{2}$ and is known as Lesser Cholistan. It is adjacent to canal irrigated region and progresses with a series of saline alluvial clayey flat land alternating with low sand dunes. The purely aeolian sandy desert, called the Greater Cholistan covers $8,130 \mathrm{Km}^{2}$ in the southern region consisting of various forms of sand ridges and inter-ridges valleys. It extends about $480 \mathrm{~km}$ in length and 32 to $192 \mathrm{~km}$ in width. Cholistan Desert presents a multifarious prototype of alluvial and aeolian depositions [20-22].

The desert of Cholistan is one of the key ecological arid zones with extreme seasonal variation and consists of a wide variety of edaphic conditions as described above. Human population of this desert comprises of more than 110,000 pastoral nomads (originally Buddhist and Sikh, but now comprising 95\% Muslims and 5\% Hindu communities). The economy of the Cholistan desert is predominantly pastoral and people have been practicing a nomadic lifestyle for centuries. Saraiki is the local language of the area.

The second study area of semi-arid nature (Pothwar/ Potohar) stretches from latitude $32^{\circ} 10-34^{\circ} 9 \mathrm{~N}$ and longitude $71^{\circ} 10-73^{\circ} 55 \mathrm{E}$ comprising of Attock (Attock, Fateh Jang, Hasan abdal, Jand, Pindi Gheb), Rawalpindi (Gujar Khan, Kahuta, Kotli sattian, Murree, Rawalpindi, Taxila), Islamabad, Jehlum (Jhelum, Pind Dadan Khan, Sohawa) and Chakwal (Chakwal, Choa Saidan Shah, Talagang) districts of the Punjab [23] and covers 23,160 sq. Km area. This land tract is bordered on the north by the Kala Chitta Range and the Margalla Hills, and on the south by the Salt Range. On the eastern side lies the River Jhelum and Indus on its west. This region varies in height from 305 to 610meters above sea level and lies in the north of Salt Range. Soanian Culture prevailed in this area evidenced by the data obtained from fossils, coins, tools, and remnants of primeval archaeological sites. Historically, notable areas of this region include two UNESCO World Heritage sites, The Rohtas Fort and Taxila [24,25]. Others include namely Rawat Fort, Pharwala Fort and Katasraj temple. The typical weather of Pothwar ranges from semi-arid to sub-humid subtropical continental [26]. The Plateau is drained by Hao and Swan rivers. Its topography is tremendously varied, consisting of ridges, troughs and basins. The large part of the Plateau has been eroded and dissected by streams. The Salt Range starts from nearby Jehlum district in the Jogi Tilla and Bakrala Ridges. It crosses the Indus near Kalabagh and extends southward into the districts of Bannu and DeraIsmaeel Khan. The average height of the range is about 671 meters and the Skaser peak is 1,525meters high. 


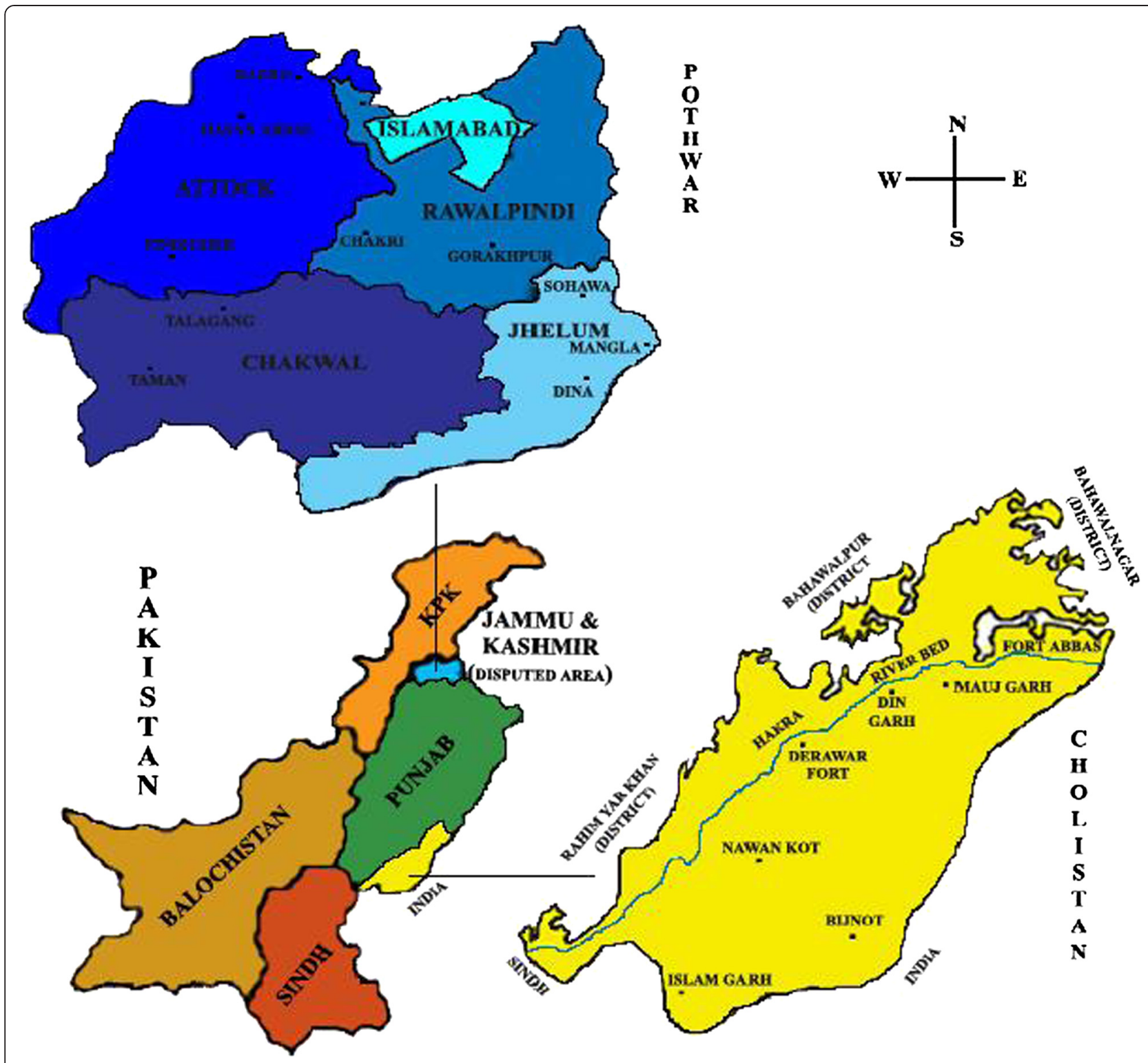

Figure $\mathbf{1}$ Location map of Pothwar and Cholistan areas in Pakistan.

Pothwar Plateau is actually an undulating, multi-colored, multi-cultural, pictorial and geologically poorly distinct area. In this densely populated land, agriculture is dependent primarily on annual rainfall (averages 380 to $510 \mathrm{~mm}$ ). Precipitation is recorded maximum in the northwest and minimum in the southwest arid zones [27]. Various soils are found in the Pothwar Plateau typically, alluvial, loess, mixed material and colluvial types resulting from sandstone and shale [26]. Inceptisols, entisols and aridisols and with traces of alfisols soils are recorded [27]. According to 1998 district census report, 7,4,64,763 people were residing in the area and there is still tremendous increase in population. Majority of the people are Muslims while minorities include Christians, Hindus and Sikhs. The urbanity level was about $40 \%$. However a great deal of inhabitants are still agrarian, many people are moving into industry and mining. Agricultural practices are dependent on rain fall [28]. Crop rotation and fallow are a common practice [29]. For instance wheat-ground nut cropping is a regular observation. Medicinal plants are mostly found as weeds which may compete with crops for natural resources [30] and at waste places.

The people in Punjab have their own distinct rural culture possessing their own principles, laws, traditions, ethnic groups Muslims (70\%), Hindus (10\%), Sikhs (15\%), and Christians (5\%), heritage, languages and much more. The rural to urban migration trend is also prevailing with many effects on traditions. The people in the rural areas 
are still highly dependent on natural products and natural remedies. They are closely related to plants and plant products play significant roles in their lives. Figure 1 shows the location map of Cholistan and Pothwar.

\section{Methods}

The study was undertaken during different seasons of the years $(2010,2011)$. Total of 136 local people including traditional healers were identified using the Participatory Rapid Appraisal Approach (PRA) [31]. PRA stipulated a valuable insight into the multiple dimensions and experiences of local people with traditional plant medicines. The informal meetings, open-ended conversations allowed us to develop problems that were important to the community but unknown to the investigators [32]. With the help of the local community the most renowned traditional healers in the study area were consulted. The traditional healers were expert practitioners who medicated the local population using ethno medicinal plants and their products. The informers were 20-80 years old including both men and women (Table 1). Informal and verbal consent was taken from each individual traditional healer who took part in the study. To confirm the use of each plant used for treating the same disease, at least three traditional healers were consulted.

The whole study area of Cholistan was divided into 11 transect lines covering almost all macro and micro habitats. The transect lines which were followed during this study were as follows: Fort Abbas to Dodhlan Plantation Border Line Area, Dak wala to Border Line Area, Fort Morot to Rana Bhana, Kalay Pahar to Border Line Area, Yazman to Wanjuhar, Yazman to Bijnot, Fort Derawar to Bijnot, Fort Derawar to Garare Wala, Fort Khair Garh to Kakki Wala, Kot Murid to Ghunyan Wala and Rahim Yar Khan to Islam Garg while in Pothwar region, villages in Chakwal, Talagang, Jhelum, Rawalpindi, Gujar Khan, Murree, Kahuta, Pindi Gheb, Attock, Kala Chitta hills were randomly selected.

Visits were made to the places where traditional healers usually accumulate plants for their therapeutic purposes. The plants mentioned in this manuscript as traditional medicines were identified in the field by the traditional healers during the dialogues between them. Voucher specimens were assigned when plants encountered for the first time. They were also assigned during flowering or fruiting. The plant specimens were treated as per standard taxonomic procedures [33]. The collected specimens

Table 1 Age and sex characteristics of traditional healers interviewed in the present study

\begin{tabular}{lllll}
\hline Gender & \multicolumn{2}{l}{ Age (years) } & & Total \\
\cline { 2 - 4 } & $\mathbf{2 0 - 4 0}$ & $\mathbf{4 0 - 6 0}$ & $\mathbf{6 0 - 8 0}$ & \\
\hline Male & 53 & 38 & 17 & 108 \\
Female & 7 & 16 & 5 & 28 \\
\hline
\end{tabular}

were dried in newspapers/blotting papers. The dried specimens were sprayed with a saturated solution of Mercuric Chloride in rectified spirit. The specimens were mounted very carefully on herbarium sheets of standard size $(41.25 \times 28.75 \mathrm{~cm})$ by using German glue. All the specimens were fully labeled on the right lower corner. The field data was then given herbarium labels. The herbarium specimens of this work have been deposited in the herbarium of Cholistan Institute of Desert Studies, The Islamia University of Bahawalpur. For small herbaceous plants, the entire plants were collected. To get insight of folklore remedies and their uses and to get first hand knowledge, meetings were held with elderly inhabitants, local Hakeems (Herbal practitioners) and domestic women of Cholistan desert and Pothwar area. The interviews and deliberations were executed in Siraki, Pothwari and Punjabi, the local languages of Cholistan and Pothwar correspondingly since the authors are native speakers of the languages. Data on specific plant parts (leaves, twigs, fruits, pods stem bark, roots etc.), and the ethno-pharmacological values were collected. For the record of medicinal uses, information on the plant parts used, their collection, processing and preparation of drugs, properties, mode of administration dosage and the diseases cured were also recorded. Collected information was also verified in different localities from local inhabitants either by showing the plant specimen or telling local names to the respondents. The plants were scientifically identified and documented as well. Scientific literature already cited was also reviewed to cross check the collected information about medicinal and ethno-botanical uses.

The descriptive data gathered for this study was recorded and saved with respect to date. Contradictory and exclusive statements were noticed and were been given close attention. The repeated data was recorded in this report by data reduction technique, the systematic content analysis. In its broad sense, different reviews have highlighted a range of aspects of content analysis, from its capacity to generate quantitative descriptions by analyzing word counts [34] to its ability to help researchers infer conclusions from a text by breaking it into distinct entities of useful data that can then be implicitly rationalized that is compressing many words of text into fewer manageable groups [35].

\section{Results and discussion}

Medicinal plant diversity

This study records 67 plant species as useful in traditionally curing 123 human diseases in Cholistan Desert (Table 2). These medicinal plants were distributed among 29 families and 55 genera. The largest proportion of medicinal plants collected belonged to the families Zygophyllaceae (5), Capparidaceae (5), Poaceae (6), 
Table 2 Medicinal Flora of Cholistan Desert (Southern Punjab)

\begin{tabular}{|c|c|c|c|c|c|c|}
\hline Sr. No. & $\begin{array}{l}\text { Plant Name [voucher } \\
\text { specimen \#] }\end{array}$ & Vernacular name & Family & Plant part used & Disease cured & References \\
\hline 1. & $\begin{array}{l}\text { Abutilon muticum (Del.ex } \\
\text { DC.) Sweet [2510/CIDS/IUB] }\end{array}$ & Kanghi-buti & Malvaceae & Leaves and roots & $\begin{array}{l}\text { Renal stones and heartburn. } \\
\text { Infectious diseases. }\end{array}$ & $\begin{array}{l}\text { Traditional Health } \\
\text { Healers }[\mathrm{THH}] ;[60]\end{array}$ \\
\hline 2. & $\begin{array}{l}\text { Acacia jacquemontii Benth. } \\
\text { [2512/CIDS/IUB] }\end{array}$ & Banwli & Mimosaceae & Bark and leaves & $\begin{array}{l}\text { Chickenpox, pain, fever and } \\
\text { small pox }\end{array}$ & {$[\mathrm{THH}]$} \\
\hline \multirow[t]{2}{*}{3.} & \multirow[t]{2}{*}{$\begin{array}{l}\text { Acacia nilotica (Linn.) Delile } \\
\text { [2513/CIDS/IUB] }\end{array}$} & \multirow[t]{2}{*}{ Babul or Kikar } & \multirow[t]{2}{*}{ Mimosaceae } & \multirow[t]{2}{*}{$\begin{array}{l}\text { Leaves, bark, } \\
\text { flowers, fruit and } \\
\text { gum }\end{array}$} & $\begin{array}{l}\text { Men sex problems, diarrhea, } \\
\text { sexual debility, hemorrhages } \\
\text { and high blood sugar. }\end{array}$ & \multirow[t]{2}{*}[\mathrm{THH}]{$;[61]$} \\
\hline & & & & & Used as tonic and cure fever. & \\
\hline \multirow[t]{2}{*}{4.} & \multirow[t]{2}{*}{$\begin{array}{l}\text { Aerva javanica var. javanica } \\
\text { [2201/CIDS/IUB] }\end{array}$} & \multirow[t]{2}{*}{ Bui } & \multirow[t]{2}{*}{ Amaranthaceae } & \multirow[t]{2}{*}{$\begin{array}{l}\text { Root, bark and } \\
\text { leaves }\end{array}$} & $\begin{array}{l}\text { Renal stones and upper- } \\
\text { respiratory infection. }\end{array}$ & \multirow[t]{2}{*}[\mathrm{THH}]{$;[62]$} \\
\hline & & & & & $\begin{array}{l}\text { Constipation and remove } \\
\text { gastrointestinal parasites. }\end{array}$ & \\
\hline \multirow[t]{2}{*}{5.} & \multirow[t]{2}{*}{$\begin{array}{l}\text { Alhagi maurorum Medic } \\
\text { [2529/CIDS/IUB] }\end{array}$} & \multirow[t]{2}{*}{ Jawansa } & \multirow[t]{2}{*}{ Papilionaceae } & \multirow[t]{2}{*}{ Whole plant } & $\begin{array}{l}\text { Blood purifier, fever, Jaundice } \\
\text { and respiratory diseases. }\end{array}$ & \multirow[t]{2}{*}[\mathrm{THH}]{$;[63]$} \\
\hline & & & & & $\begin{array}{l}\text { Increased perspiration, cough, } \\
\text { and constipation. }\end{array}$ & \\
\hline 6. & $\begin{array}{l}\text { Blepharis scindica T. Anders. } \\
\text { [2001/CIDS/IUB] }\end{array}$ & Gandi-boti & Acanthaceae & Whole plant & General weakness and pain & {$[\mathrm{THH}]$} \\
\hline \multirow[t]{2}{*}{7.} & \multirow[t]{2}{*}{$\begin{array}{l}\text { Boerhavia procumbens } \\
\text { Banks ex Roxb [2518/ } \\
\text { CIDS/IUB] }\end{array}$} & \multirow[t]{2}{*}{ Biskhipra } & \multirow[t]{2}{*}{ Nyctaginaceae } & \multirow[t]{2}{*}{ Whole plant } & $\begin{array}{l}\text { Chest infections, renal failure } \\
\text { and painful periods in } \\
\text { women. }\end{array}$ & \multirow[t]{2}{*}{$\begin{array}{l}\text { [THH]; [64]; [65]; } \\
{[66]}\end{array}$} \\
\hline & & & & & $\begin{array}{l}\text { Blood purifier. Liver diseases, } \\
\text { sexually transmitted } \\
\text { infections and sluggishness. }\end{array}$ & \\
\hline \multirow[t]{2}{*}{8.} & \multirow{2}{*}{$\begin{array}{l}\text { Calligonum polygonoides } \\
\text { Linn [2663/CIDS/IUB] }\end{array}$} & \multirow[t]{2}{*}{ Phog } & \multirow[t]{2}{*}{ Polygonaceae } & \multirow{2}{*}{$\begin{array}{l}\text { Flowers and green } \\
\text { twigs }\end{array}$} & Sore eyes, severe thirst. & {$[\mathrm{THH}]$} \\
\hline & & & & & $\begin{array}{l}\text { Indigestion, sore throat and } \\
\text { pain. }\end{array}$ & \\
\hline 9. & $\begin{array}{l}\text { Calotropis procera subsp. } \\
\text { hamiltonii (Wight) Ali } \\
\text { [2005/CIDS/IUB] }\end{array}$ & Ak & Asclepiadaceae & $\begin{array}{l}\text { Latex, leaves, } \\
\text { flower buds and } \\
\text { root bark }\end{array}$ & $\begin{array}{l}\text { Painful menstruation, uterus } \\
\text { problems, asthma, } \\
\text { stomachache and muscular } \\
\text { weakness. Snake bite, piles, } \\
\text { leprosy, sexually transmitted } \\
\text { diseases, asthma and joint } \\
\text { pain. }\end{array}$ & {$[\mathrm{THH}] ;[67] ;[68]$} \\
\hline 10. & $\begin{array}{l}\text { Capparis decidua (Forsskal.) } \\
\text { Edgew [2315/CIDS/IUB] }\end{array}$ & Karir & Capparidaceae & $\begin{array}{l}\text { Leaves, fruit and } \\
\text { stem }\end{array}$ & $\begin{array}{l}\text { Piles, fevers, painful } \\
\text { menstruation, high blood } \\
\text { sugar, obesity, indigestion } \\
\text { and bone fractures. }\end{array}$ & {$[\mathrm{THH}] ;[69]$} \\
\hline & & & & & $\begin{array}{l}\text { Remove intestinal worms, } \\
\text { used as sexual stimulant, } \\
\text { relieves flatulence, increased } \\
\text { perspiration and constipation. }\end{array}$ & \\
\hline 11. & $\begin{array}{l}\text { Capparis spinosa Linn. } \\
\text { [2316/CIDS/IUB] }\end{array}$ & Kubber & Capparidaceae & Leaves flowers & $\begin{array}{l}\text { Joint, muscle pain, asthma, } \\
\text { indigestion and liver } \\
\text { disorders }\end{array}$ & {$[\mathrm{THH}]$} \\
\hline 12. & $\begin{array}{l}\text { Cassia italica subsp. Italica } \\
\text { [2314/CIDS/IUB] }\end{array}$ & $\begin{array}{l}\text { Ghoray-wall or } \\
\text { Sana }\end{array}$ & Caesalpinaceae & Whole plant & $\begin{array}{l}\text { Arthritis, pain, fevers and } \\
\text { indigestion }\end{array}$ & {$[\mathrm{THH}]$} \\
\hline 13. & $\begin{array}{l}\text { Cenchrus biflorus Roxb. } \\
\text { [2531/CIDS/IUB] }\end{array}$ & Mohabat Boti & Poaceae & Whole plant & Kills intestinal worms & {$[\mathrm{THH}]$} \\
\hline 14. & $\begin{array}{l}\text { Cenchrus ciliaris L. } \\
\text { [2530/CIDS/IUB] }\end{array}$ & Daman & Poaceae & Whole plant & $\begin{array}{l}\text { Intestinal worms, increase } \\
\text { milk production in cattle and } \\
\text { wound healing. }\end{array}$ & {$[\mathrm{THH}] ;[\mathrm{70}]$} \\
\hline & & & & & $\begin{array}{l}\text { Relieves pain and used as } \\
\text { emollient. }\end{array}$ & \\
\hline
\end{tabular}


Table 2 Medicinal Flora of Cholistan Desert (Southern Punjab) (Continued)

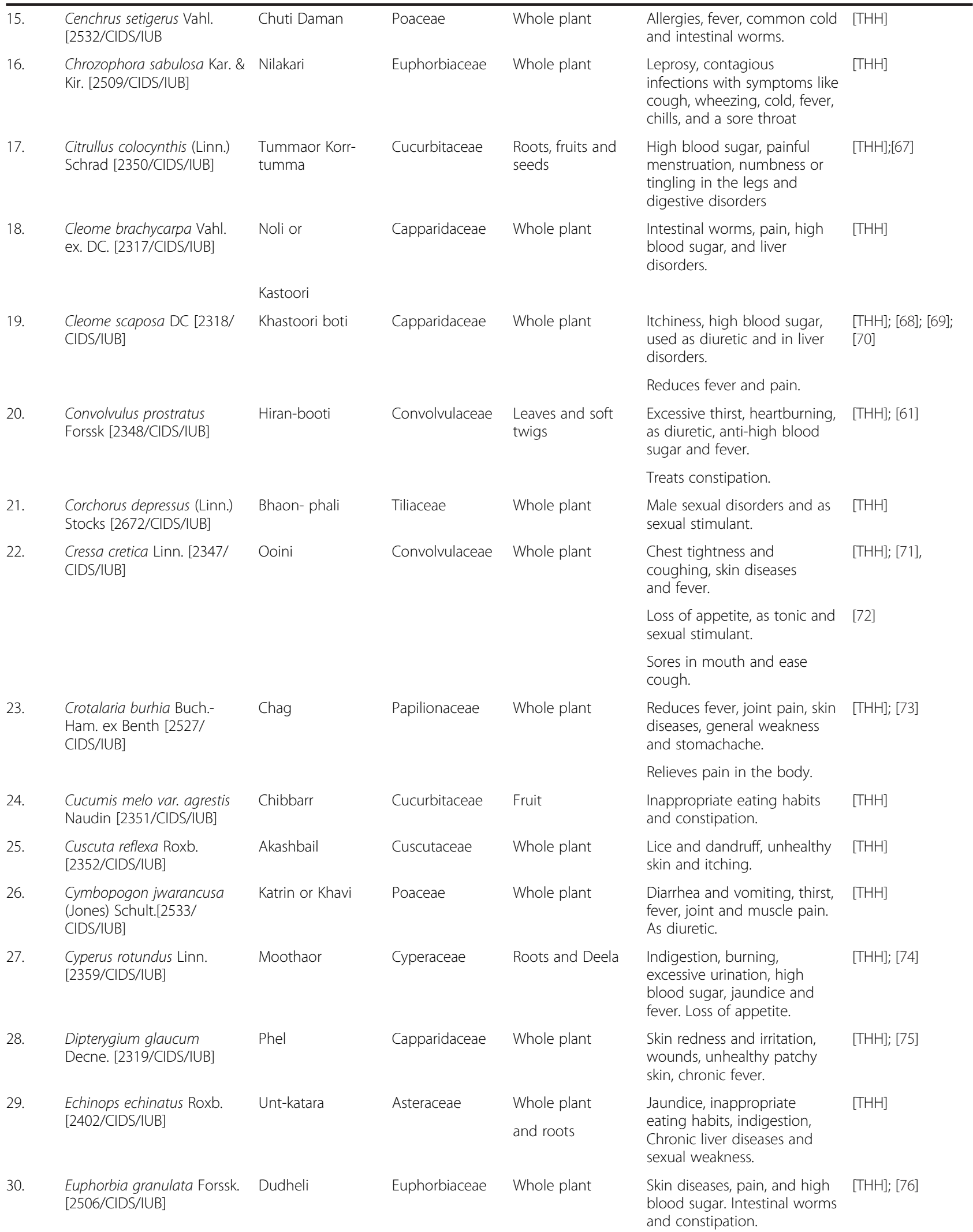


Table 2 Medicinal Flora of Cholistan Desert (Southern Punjab) (Continued)

\begin{tabular}{|c|c|c|c|c|c|c|}
\hline 31. & $\begin{array}{l}\text { Euphorbia prostrata Ait. } \\
\text { [2503/CIDS/IUB] }\end{array}$ & Hazar-dani & Euphorbiaceae & Whole plant & $\begin{array}{l}\text { Piles, sexual weakness, skin } \\
\text { redness and irritation } \\
\text { and pain. }\end{array}$ & {$[\mathrm{THH}] ;[76]$} \\
\hline 32. & $\begin{array}{l}\text { Fagonia cretica Linn [2675/ } \\
\text { CIDS/IUB] }\end{array}$ & Dhmasa & Zygophyllaceae & Whole plant & $\begin{array}{l}\text { Liver diseases, lack of blood, } \\
\text { fever, pain and as blood } \\
\text { purifier and increase stamina. }\end{array}$ & $(\mathrm{THH})$ \\
\hline \multirow[t]{2}{*}{33.} & $\begin{array}{l}\text { Farsetia hamiltonii Royle } \\
\text { [2313/CIDS/IUB] }\end{array}$ & Lathi or & Brassicaceae & Whole plant & $\begin{array}{l}\text { As tonic and against } \\
\text { stomachache and joint, } \\
\text { muscle pain and diabetes }\end{array}$ & {$[\mathrm{THH}] ;[77]$} \\
\hline & & Farid- booti & & & & \\
\hline \multirow[t]{2}{*}{34.} & $\begin{array}{l}\text { Gisekia pharnaceoides } \\
\text { Linn.[2101/CIDS/IUB] }\end{array}$ & Buloka-sag & Aizoaceae & Whole plant & $\begin{array}{l}\text { Jaundice, inappropriate } \\
\text { eating habits, fever and pain. }\end{array}$ & {$[\mathrm{THH}] ;[78]$} \\
\hline & & & & & $\begin{array}{l}\text { Constipation, remove } \\
\text { intestinal worms and skin } \\
\text { infections. }\end{array}$ & \\
\hline 35. & $\begin{array}{l}\text { Glinus lotoides Linn [2514/ } \\
\text { CIDS/IUB] }\end{array}$ & Gandi-booti & Molluginaceae & Whole plant & $\begin{array}{l}\text { Diarrhea, poor functioning of } \\
\text { liver, externally to cure boils } \\
\text { and wounds. }\end{array}$ & {$[\mathrm{THH}]$} \\
\hline 36. & $\begin{array}{l}\text { Grewia villosa Willd. [2673/ } \\
\text { CIDS/IUB] }\end{array}$ & Jalidar & Tiliaceae & Whole plant & $\begin{array}{l}\text { Urinary tract infections, liver } \\
\text { diseases, eye-ache, sexually } \\
\text { transmitting diseases. }\end{array}$ & {$[\mathrm{THH}]$} \\
\hline \multirow[t]{2}{*}{37.} & $\begin{array}{l}\text { Haloxylon recurvum Bunge. } \\
\text { ex. Boiss. [2335/CIDS/IUB] }\end{array}$ & Khar or & Chenopodiaceae & Whole plant & $\begin{array}{l}\text { Gastric problems and kidney } \\
\text { Stones. }\end{array}$ & {$[\mathrm{THH}]$} \\
\hline & & Sajji & & & & \\
\hline \multirow[t]{2}{*}{38.} & $\begin{array}{l}\text { Haloxylon salicornicum } \\
\text { (Moq.) Bunge [2336/ } \\
\text { CIDS/IUB] }\end{array}$ & Lana & Chenopodiaceae & Whole plant & $\begin{array}{l}\text { Bleeding gums, Indigestion } \\
\text { and insect stings. Prevent } \\
\text { damage to the liver. }\end{array}$ & {$[\mathrm{THH}]$} \\
\hline & & & & & & {$[79]$} \\
\hline 39. & $\begin{array}{l}\text { Heliotropium crispum Desf. } \\
\text { [2321/CIDS/IUB] }\end{array}$ & Kali-lani & Boraginaceae & Whole plant & $\begin{array}{l}\text { Weakness, indigestion and } \\
\text { laziness. }\end{array}$ & {$[\mathrm{THH}] ;[62]$} \\
\hline 40. & $\begin{array}{l}\text { Heliotropium strigosum } \\
\text { subsp. Strigosum } \\
\text { [2311/CIDS/IUB] }\end{array}$ & Gorakh-Pan & Boraginaceae & Whole plant & $\begin{array}{l}\text { Sore eyes, open wounds, } \\
\text { sore throat, sore nipples of } \\
\text { breasts, jaundice, used as } \\
\text { blood purifier and to cure } \\
\text { severe cough. }\end{array}$ & {$[\mathrm{THH}]$} \\
\hline 41. & $\begin{array}{l}\text { Indigofera argentea Burm. } \\
\text { f. [2528/CIDS/IUB] }\end{array}$ & Neel & Papilionaceae & Whole plant & $\begin{array}{l}\text { Intestinal parasites and } \\
\text { patchy skin. }\end{array}$ & {$[\mathrm{THH}]$} \\
\hline 42. & $\begin{array}{l}\text { Launaea nudicaulis Less } \\
\text { [2403/CIDS/IUB] }\end{array}$ & Dudhkal & Asteraceae & Whole plant & $\begin{array}{l}\text { Chronic constipation and } \\
\text { Indigestion. }\end{array}$ & {$[\mathrm{THH}]$} \\
\hline 43. & $\begin{array}{l}\text { Leptadenia pyrotechnica } \\
\text { (Forsskal.) Decne [2002/ } \\
\text { CIDS/IUB] }\end{array}$ & Khip & Asclepiadaceae & Leaves and shoots & $\begin{array}{l}\text { Abdominal cramps, } \\
\text { constipation, painful } \\
\text { menstruation, obesity and } \\
\text { high blood sugar. }\end{array}$ & {$[\mathrm{THH}]$} \\
\hline \multirow[t]{2}{*}{44.} & $\begin{array}{l}\text { Mollugo cerviana (L.) } \\
\text { Seringe [2515/CIDS/IUB] }\end{array}$ & Padi or & Molluginaceae & Roots & $\begin{array}{l}\text { Fever, burning urination and } \\
\text { sexually transmitted diseases. }\end{array}$ & {$[\mathrm{THH}] ;[80] ;$} \\
\hline & & Sarr & & & $\begin{array}{l}\text { Skin itching and as blood } \\
\text { purifier. }\end{array}$ & {$[64]$} \\
\hline 45. & $\begin{array}{l}\text { Mollugo nudicaulis Lamk. } \\
\text { [2516/CIDS/IUB] }\end{array}$ & Gandi-buti & Molluginaceae & Whole plant & $\begin{array}{l}\text { Chest infection, whooping } \\
\text { cough; leaves are applied as } \\
\text { poultice on wounds and } \\
\text { boils. }\end{array}$ & {$[\mathrm{THH}]$} \\
\hline 46. & $\begin{array}{l}\text { Mukia maderaspatana } \\
\text { (Linn.) M.J. Roem [2349/ } \\
\text { CIDS/IUB] }\end{array}$ & Gawala-kakri & Cucurbitaceae & $\begin{array}{l}\text { Shoots, roots and } \\
\text { seeds }\end{array}$ & $\begin{array}{l}\text { Jaundice, fever, muscular } \\
\text { weakness and lower back } \\
\text { pain. }\end{array}$ & {$[\mathrm{THH}]$} \\
\hline 47. & $\begin{array}{l}\text { Neurada procumbens Linn } \\
\text { [2517/CIDS/IUB] }\end{array}$ & Chhapri & Neuradaceae & Whole plant & $\begin{array}{l}\text { As sexual tonic and cure } \\
\text { general weakness. }\end{array}$ & {$[\mathrm{THH}] ;[62]$} \\
\hline
\end{tabular}


Table 2 Medicinal Flora of Cholistan Desert (Southern Punjab) (Continued)

\begin{tabular}{|c|c|c|c|c|c|c|}
\hline 48. & $\begin{array}{l}\text { Oligochaeta ramosa (Roxb.) } \\
\text { Magenitz [2401/CIDS/IUB] }\end{array}$ & Birham dandi & Asteraceae & Whole plant & $\begin{array}{l}\text { Irritation, liver diseases, joint } \\
\text { pain and as brain tonic }\end{array}$ & {$[\mathrm{THH}]$} \\
\hline 49. & $\begin{array}{l}\text { Oxystelma esculentum (Linn. } \\
\text { f.) R. Brown [2003/CIDS/IUB] }\end{array}$ & Dudhani & Asclepiadaceae & Whole plant & $\begin{array}{l}\text { Painful urination, sexually } \\
\text { transmitted diseases, acne } \\
\text { and pain. }\end{array}$ & {$[\mathrm{THH}]$} \\
\hline 50. & $\begin{array}{l}\text { Panicum antidotale Retz } \\
\text { [2534/CIDS/IUB] }\end{array}$ & $\begin{array}{l}\text { Murrot or Bansi } \\
\text { ghaa }\end{array}$ & Poaceae & Whole plant & $\begin{array}{l}\text { Severe sore throat, small pox } \\
\text { and respiratory tract infection }\end{array}$ & {$[\mathrm{THH}]$} \\
\hline 51. & $\begin{array}{l}\text { Peganum harmala Linn. } \\
\text { [2678/CIDS/IUB] }\end{array}$ & Harmal & Zygophyllaceae & $\begin{array}{l}\text { Leaves, roots and } \\
\text { seeds }\end{array}$ & $\begin{array}{l}\text { Emotional disturbances, } \\
\text { painful menstruation, } \\
\text { seizures, insanity and itchy } \\
\text { skin. Abdominal pain and } \\
\text { smoke has insecticidal } \\
\text { properties. }\end{array}$ & {$[\mathrm{THH}] ;[67]$} \\
\hline 52. & $\begin{array}{l}\text { Pergularia daemia (Jacq.) N. } \\
\text { E. Brown. [2004/CIDS/IUB] }\end{array}$ & Karial & Asclepiadaceae & Whole plant & $\begin{array}{l}\text { Intestinal worms, reduces } \\
\text { fever, flatulence, chest } \\
\text { tightness, stomachache and } \\
\text { gynecological problems }\end{array}$ & {$[\mathrm{THH}]$} \\
\hline \multirow[t]{2}{*}{53.} & $\begin{array}{l}\text { Polygonum plebejum R. } \\
\mathrm{Br}[2538 / \mathrm{CIDS} / \mathrm{IUB}]\end{array}$ & Chiri- & Polygonaceae & $\begin{array}{l}\text { Roots and whole } \\
\text { plant }\end{array}$ & $\begin{array}{l}\text { Respiratory tract infections, } \\
\text { indigestion, vomiting and } \\
\text { diarrhea }\end{array}$ & {$[\mathrm{THH}]$} \\
\hline & & Hatta & & & & \\
\hline 54. & $\begin{array}{l}\text { Prosopis cineraria (Linn.) } \\
\text { Druce [2511/CIDS/IUB] }\end{array}$ & $\begin{array}{l}\text { Jandi or Jand or } \\
\text { kunda }\end{array}$ & Mimosaceae & $\begin{array}{l}\text { Leaves, bark, } \\
\text { flowers and pods }\end{array}$ & $\begin{array}{l}\text { Heal wounds, for birth } \\
\text { control, blood deficiency, } \\
\text { protein deficiency, painful } \\
\text { menstruation and joint, } \\
\text { muscle pain. }\end{array}$ & {$[\mathrm{THH}]$} \\
\hline 55. & $\begin{array}{l}\text { Pulicaria crispa (Cass.) } \\
\text { Benth. \& Hook. f. [2339/ } \\
\text { CIDS/IUB] }\end{array}$ & Bui & Compositae & Whole plant & $\begin{array}{l}\text { Fever, headache, severe cold, } \\
\text { cough and jaundice. }\end{array}$ & {$[\mathrm{THH}]$} \\
\hline 56. & $\begin{array}{l}\text { Salsola baryosma (Roem. } \\
\text { ex. Scult.) Dany. [2337/ } \\
\text { CIDS/IUB] }\end{array}$ & Lani & Chenopodiaceae & Whole plant & $\begin{array}{l}\text { Remove intestinal worms, } \\
\text { itching, indigestion, and } \\
\text { sores. }\end{array}$ & {$[\mathrm{THH}]$} \\
\hline 57. & $\begin{array}{l}\text { Salvadora oleoides Decne. } \\
\text { [2667/CIDS/IUB] }\end{array}$ & Pilu & Salvadoraceae & $\begin{array}{l}\text { Fruit, bark and } \\
\text { leaves }\end{array}$ & $\begin{array}{l}\text { Nutritive deficiency, } \\
\text { inappropriate eating habits, } \\
\text { skin boils, high blood sugar, } \\
\text { gum bleeding and } \\
\text { stomachache. }\end{array}$ & {$[\mathrm{THH}]$} \\
\hline 58. & $\begin{array}{l}\text { Solanum surattense Burm. } \\
\text { F. [2668/CIDS/IUB] }\end{array}$ & Kanderi & Solanaceae & Whole plant & $\begin{array}{l}\text { Joint pain, Fever, used as } \\
\text { blood purifier, in breathing } \\
\text { problems, severe headache, } \\
\text { leprosy, as diuretic, hair tonic } \\
\text { and cure abdomen pain, gas } \\
\text { trouble, chronic cough and } \\
\text { pain. }\end{array}$ & {$[\mathrm{THH}] ;[36] ;[67]$} \\
\hline 59. & $\begin{array}{l}\text { Sporobolus ioclados (Nees } \\
\text { ex Trin.) Nees [2535/ } \\
\text { CIDS/IUB] }\end{array}$ & & Poaceae & Whole plant & $\begin{array}{l}\text { Severe fever, headache and } \\
\text { vomiting. }\end{array}$ & {$[\mathrm{THH}]$} \\
\hline \multirow[t]{2}{*}{60.} & $\begin{array}{l}\text { Suaeda fruticosa Forssk. ex } \\
\text { J. F. Gmelin [2338/CIDS/IUB] }\end{array}$ & Kali lani & Chenopodiaceae & Whole plant & $\begin{array}{l}\text { Constipation, painful } \\
\text { menstruation, red eyes, } \\
\text { indigestion and wound } \\
\text { healing. }\end{array}$ & {$[\mathrm{THH}]$} \\
\hline & & & & & & {$[81]$} \\
\hline 61. & $\begin{array}{l}\text { Tamarix aphylla (Linn.) } \\
\text { Karst [2671/CIDS/IUB] }\end{array}$ & $\begin{array}{l}\text { Jhao and Ukan or } \\
\text { Frash }\end{array}$ & Tamaracaceae & $\begin{array}{l}\text { Leaves, bark and } \\
\text { nuts }\end{array}$ & $\begin{array}{l}\text { Liver diseases, indigestion, } \\
\text { stomachache, leucorrhoea, } \\
\text { sexual weakness and skin } \\
\text { problems. }\end{array}$ & {$[\mathrm{THH}]$} \\
\hline 62. & $\begin{array}{l}\text { Tribulus longipetalus } \\
\text { subsp. Longipetalus } \\
\text { [2676/CIDS/IUB] }\end{array}$ & Tirkandi or & Zygophyllaceae & Fruits and seeds & $\begin{array}{l}\text { Renal stones, male sexual } \\
\text { problems, anemia and } \\
\text { general weakness. }\end{array}$ & {$[\mathrm{THH}]$} \\
\hline
\end{tabular}


Table 2 Medicinal Flora of Cholistan Desert (Southern Punjab) (Continued)

\begin{tabular}{|c|c|c|c|c|c|c|}
\hline & & Bakharra & & & & \\
\hline \multirow[t]{2}{*}{63.} & \multirow{2}{*}{$\begin{array}{l}\text { Tribulus longipetalus subsp. } \\
\text { Macropterus [2677/ } \\
\text { CIDS/IUB] }\end{array}$} & Tirkindi or & \multirow[t]{2}{*}{ Zygophyllaceae } & \multirow[t]{2}{*}{ Whole plant } & \multirow{2}{*}{$\begin{array}{l}\text { Male sexual problems, itchy } \\
\text { skin, chest/heart pain, piles, } \\
\text { bleeding from nose and pain. }\end{array}$} & \multirow[t]{2}{*}[\mathrm{THH}]{} \\
\hline & & Bakharra & & & & \\
\hline 64. & $\begin{array}{l}\text { Withania coagulens (Stocks) } \\
\text { Dunal }[2669 / \text { CIDS/IUB] }\end{array}$ & Paneer & Solanaceae & $\begin{array}{l}\text { Whole plant and } \\
\text { fruit }\end{array}$ & $\begin{array}{l}\text { Jaundice, inappropriate } \\
\text { eating habits and skin } \\
\text { problems. }\end{array}$ & {$[\mathrm{THH}]$} \\
\hline 65. & $\begin{array}{l}\text { Withania somnifera (Linn.) } \\
\text { Dunal [2670/CIDS/IUB] }\end{array}$ & Asgandh & Solanaceae & $\begin{array}{l}\text { Leaves and root } \\
\text { bark }\end{array}$ & $\begin{array}{l}\text { Boils, nerve weakness, joint } \\
\text { pain and as sexual stimulant. }\end{array}$ & [THH]; [36]; [67] \\
\hline 66. & $\begin{array}{l}\text { Ziziphus nummularia } \\
\text { (Burm. f.) Wight \& Arn } \\
\text { [2664/CIDS/IUB] }\end{array}$ & Beri & Rhamnaceae & $\begin{array}{l}\text { Fruit, bark, leaves } \\
\text { and seeds }\end{array}$ & $\begin{array}{l}\text { Skin diseases, cold, cough, } \\
\text { stomachache, diarrhea, hair } \\
\text { roughness, high blood sugar } \\
\text { and help wound healing. }\end{array}$ & $\begin{array}{l}{[\mathrm{THH}] ;[36] ;[67] ;} \\
{[82]}\end{array}$ \\
\hline 67. & $\begin{array}{l}\text { Zygophyllum simplex Linn } \\
\text { [2674/CIDS/IUB] }\end{array}$ & Lunak & Zygophyllaceae & Whole plant & $\begin{array}{l}\text { Patchy skin, wounds, acne } \\
\text { and Bleeding }\end{array}$ & {$[\mathrm{THH}]$} \\
\hline
\end{tabular}

Solanaceae (3), Asclepiadaceae (4), Cucurbitaceae (3), Chenopodiaceae (4), Papilionaceae (3), Molluginaceae (3), Euphorbiaceae (3), Asteraceae (3) and Mimosaceae (3). Poaceae, Zygophyllaceae and Capparidaceae constitute the maximum diversity of species used in herbal medication.

Cholistan Desert is uniquely located in wild land with dearth of endemic flora counting only 128 species belonging to 32 families. During the present study people including local elders (Siana), herbal and homoeopathic practitioners and spiritual healers were interviewed. They play an imperative role in primary healthcare of the local inhabitants as the majority of their clients come from poor families who cannot meet the expense of the modern healthcare services. As said by traditional healers, the local people are still dependent on wild plants for prime healthcare owing to the widespread faith in its efficiency. According to the current survey, local people for curing various diseases, commonly use 67 plant species belonging to 29 families. The diseases cured vary from simple stomachache to more complicated such as male and female urino-genital disorders. It is evident from Table 2 that 14 plant species are being used for the treatment of gastrointestinal tract disorders. Moreover, it is observed that 16 plant species are consumed as antibacterial and cure for skin diseases. 10 of the plant species are particularly utilized for respiratory tract problems, whereas, for musculoskeletal and joint disorders 10 plant species are used. There are 5 species being consumed for the male sexual disorders, and 10 species for the female sexual disorders. For urinary tract infections 5 plant species have been exploited, and 10 plant species are being consumed as anti-diabetics. In addition to this, traditional healers are using 14 plant species to cure fever, 7 plant species to cure liver diseases, 9 plant species to treat jaundice and renal stones are being cured with 6 plant species. Five plants including Heliotropium strigosum, Withania somnifera, Mukia maderaspatana, Cymbopogon jwarancusa, and Peganum harmala are commonly used for the treatment of CNS disorders, like dementia.

Data acquired from Northern Punjab (Pothwar area) is assembled in Table 3 and the plants species are sorted alphabetically. A sum of 86 plant species belonging to 38 families have been reported, used for the cure of different diseases. The highest numbers (8) of medicinal plants are from Asteraceae. 22 are found to be used in treatment of jaundice and liver diseases. 22 plant species have been benefited as anti-diabetic. Solanum surratense has been used by local people for the cure of abdomen pain, gas trouble and chronic coughs and pain. It is also reported to possess antibacterial activity against drinking water bacteria $[36,37]$. Four plant species are used to cure sexually transmitted diseases, two plant species to treat sexual impotency in males, 3 being used for treatment of rheumatic/ joint pain, 7 for cough and asthma, 3 against piles, 2 species to cure urinary problems, 5 used in cure to skin problems and dandruff, 4 species for eye diseases, 4 for high blood pressure treatment, 20 for digestive system disorders, rest of the reported plants species are used against various other ailments like fever, ear pain, touch ache, few as antidote etc. In district Attock, 35 different types of human ailments that have been reported in previous studies to be used in conventional system [10].

Data acquired from Northern Punjab (Pothwar area) and Southern Punjab (Cholistan) bears only $10.5 \%$ similarity. The similar medicinal plants of both areas are comprised of 9 plant species such as Calotropis procera subsp. hamiltonii (Wight) Ali, Citrullus colocynthis (Linn.) Schrad, Cyperus rotundus Linn., Acacia nilotica (Linn.) Delile, Boerhavia procumbens Banks ex Roxb, Ziziphus nummularia (Burm. f.) Wight \& Arn., Solanum surattense Burm. F., Withania somnifera (Linn.) Dunal and Peganum harmala Linn. 
Table 3 Medicinal Flora of Pothwar Plateau (Northern Punjab)

\begin{tabular}{|c|c|c|c|c|c|c|}
\hline Sr. No. & $\begin{array}{l}\text { Plant Name [voucher } \\
\text { specimen \#] }\end{array}$ & $\begin{array}{l}\text { Vernacular } \\
\text { name }\end{array}$ & Family & Plant part used & Disease cure & References \\
\hline 1 & $\begin{array}{l}\text { Abutilon indicum G. Don } \\
\text { [0001/ASAB/NUST] }\end{array}$ & Kanghi & Malvaceae & Whole plant & $\begin{array}{l}\text { Diarrhea, sexually transmitted } \\
\text { diseases, burning with } \\
\text { urination }\end{array}$ & $\begin{array}{l}\text { Traditional Health } \\
\text { Healers }[\mathrm{THH}] ;[65]\end{array}$ \\
\hline 2 & $\begin{array}{l}\text { Acacia modesta Wall. } \\
\text { [0002/ASAB/NUST] }\end{array}$ & Phulahi & Mimosaceae & Bark of tree & $\begin{array}{l}\text { gas trouble and abdominal } \\
\text { diseases }\end{array}$ & {$[\mathrm{THH}] ;[67]$} \\
\hline 3 & $\begin{array}{l}\text { Acacia nilotica (L.) Delile. } \\
\text { [0003/ASAB/NUST] }\end{array}$ & Kiker & Mimosaceae & $\begin{array}{l}\text { Bark, leaves and } \\
\text { branches }\end{array}$ & $\begin{array}{l}\text { Mouth sores, gum pain and } \\
\text { toothache, eye sores, sexual } \\
\text { disability, diarrhea, asthma }\end{array}$ & {$[\mathrm{THH}] ;[67] ;[68]$} \\
\hline 4 & $\begin{array}{l}\text { Achyranthus aspera L. } \\
\text { [0004/ASAB/NUST] }\end{array}$ & Puth kanda & Amaranthaceae & Whole plant & $\begin{array}{l}\text { Excessive menstruation, piles, } \\
\text { abdominal pain, toothache, } \\
\text { severe diarrhea. }\end{array}$ & {$[\mathrm{THH}] ;[65]$} \\
\hline 5 & $\begin{array}{l}\text { Adhatoda vasica Nees } \\
\text { [0005/ASAB/NUST] }\end{array}$ & Bekkar & Acanthaceae & Leaves & High blood sugar & {$[\mathrm{THH}] ;[82]$} \\
\hline 6 & $\begin{array}{l}\text { Adiantum capillus veneris } \\
\text { L. [0006/ASAB/NUST] }\end{array}$ & Sarhaj & Adiantaceae & Leaves & Jaundice and liver diseases & {$[\mathrm{THH}] ;[83]$} \\
\hline 7 & $\begin{array}{l}\text { Albizzia lebbek (L.) Benth } \\
\text { [0007/ASAB/NUST] }\end{array}$ & Shrin & Mimosaceae & Leaves & Eye problems & {$[\mathrm{THH}] ;[67]$} \\
\hline 8 & $\begin{array}{l}\text { Allium cepa L. [0008/ } \\
\text { ASAB/NUST] }\end{array}$ & Piaz & Liliaceae & bulb & $\begin{array}{l}\text { High blood pressure, high } \\
\text { blood sugar }\end{array}$ & {$[\mathrm{THH}] ;[82]$} \\
\hline 9 & $\begin{array}{l}\text { Allium sativum L. } \\
\text { [0009/ASAB/NUST] }\end{array}$ & Thoom & Liliaceae & bulb & $\begin{array}{l}\text { Ear pain, hypertension, high } \\
\text { blood sugar }\end{array}$ & {$[\mathrm{THH}] ;[67] ;[82]$} \\
\hline 10 & $\begin{array}{l}\text { Aloe vera L. [0010/ } \\
\text { ASAB/NUST] }\end{array}$ & Knwar gandal & Liliaceae & Leaf sap, stem & $\begin{array}{l}\text { Abdominal pains, } \\
\text { constipation, skin diseases, } \\
\text { high blood sugar }\end{array}$ & {$[\mathrm{THH}] ;[67] ;[82]$} \\
\hline 11 & $\begin{array}{l}\text { Amaranthus viridis L. } \\
\text { [0011/ASAB/NUST] }\end{array}$ & Chaulai & Amaranthaceae & Leaves & $\begin{array}{l}\text { Menstrual disturbance, } \\
\text { constipation }\end{array}$ & {$[\mathrm{THH}] ;[36] ;[65]$} \\
\hline 12 & $\begin{array}{l}\text { Anethum graveolense L. } \\
\text { [0012/ASAB/NUST] }\end{array}$ & Soye & Apiaceae & seeds & Abdominal pain & {$[\mathrm{THH}] ;[67]$} \\
\hline \multirow[t]{2}{*}{13} & \multirow{2}{*}{$\begin{array}{l}\text { Argyrolobium roseum } \\
\text { (Comb) Jaub \& Spach. } \\
\text { [0013/ASAB/NUST] }\end{array}$} & Makhni & \multirow[t]{2}{*}{ Papilionaceae } & \multirow[t]{2}{*}{ Whole plant } & \multirow[t]{2}{*}{ Jaundice and liver diseases } & \multirow[t]{2}{*}[\mathrm{THH}]{$;[83]$} \\
\hline & & Booti & & & & \\
\hline 14 & $\begin{array}{l}\text { Artemisia scoparia Walds \& } \\
\text { Kit. [0014/ASAB/NUST] }\end{array}$ & $\begin{array}{l}\text { Done Jhan, } \\
\text { pinche }\end{array}$ & Asteraceae & Whole plant & Abdominal disorder, earache & {$[65]$} \\
\hline 15 & $\begin{array}{l}\text { Atriplex spp. [0015/ASAB/ } \\
\text { NUST] }\end{array}$ & Gerukh pari & Chenopodiaceae & $\begin{array}{l}\text { Leaves, whole } \\
\text { plant }\end{array}$ & $\begin{array}{l}\text { Fever, jaundice, slugishness, } \\
\text { liver disease, joint pain }\end{array}$ & {$[36]$} \\
\hline 16 & $\begin{array}{l}\text { Berberis lycium Royle. [0016/ } \\
\text { ASAB/NUST] }\end{array}$ & Sumbul & Berberidaceae & Leaves & Jaundice and liver diseases & {$[\mathrm{THH}] ;[83]$} \\
\hline 17 & $\begin{array}{l}\text { Boerhaavia procumbens } \mathrm{L} . \\
\text { [0017/ASAB/NUST] }\end{array}$ & Itsit & Nyctaginaceae & Whole plant & $\begin{array}{l}\text { Jaundice, liver diseases, } \\
\text { sexually transmitted diseases, } \\
\text { weakness }\end{array}$ & [THH]; [83]; [65] \\
\hline 18 & $\begin{array}{l}\text { Bryophyllum pinnatum Kurz. } \\
\text { [0018/ASAB/NUST] }\end{array}$ & Zakhm-e-hayat & Crassulaceae & Leaves & Wounds healing & {$[\mathrm{THH}] ;[67]$} \\
\hline 19 & $\begin{array}{l}\text { Cajanus cajan (L.) Millsp. } \\
\text { [0019/ASAB/NUST] }\end{array}$ & Arar ke dal & Papilionaceae & seeds & High blood sugar & {$[\mathrm{THH}] ;[82]$} \\
\hline 20 & $\begin{array}{l}\text { Calotropis procera Alton. F. } \\
\text { [0020/ASAB/NUST] }\end{array}$ & Ak & Asclepiadaceae & $\begin{array}{l}\text { Leaves, latex and } \\
\text { flowers }\end{array}$ & $\begin{array}{l}\text { Snake bite, piles, leprosy, } \\
\text { sexually transmitted diseases, } \\
\text { asthma, joint pain }\end{array}$ & {$[\mathrm{THH}] ;[67] ;[68]$} \\
\hline 21 & $\begin{array}{l}\text { Cannabis sativa L. } \\
\text { [0021/ASAB/NUST] }\end{array}$ & Bhang & Cannabinaceae & Leaves, flowers & $\begin{array}{l}\text { Indigestion, sexually } \\
\text { transmitted diseases, also } \\
\text { used as sedative, narcotic } \\
\text { and antidote against poison }\end{array}$ & [THH]; [36]; [65] \\
\hline 22 & $\begin{array}{l}\text { Caralluma edulis (L.) Benth } \\
\text { ex Hook. f. [0022/ASAB/ } \\
\text { NUST] }\end{array}$ & Choung & Asclepidaceae & aerial parts & High blood sugar & {$[82]$} \\
\hline
\end{tabular}


Table 3 Medicinal Flora of Pothwar Plateau (Northern Punjab) (Continued)

\begin{tabular}{|c|c|c|c|c|c|c|}
\hline 23 & $\begin{array}{l}\text { Carissa opaca Stapf. ex. } \\
\text { Haines [0023/ASAB/NUST] }\end{array}$ & Garanda & Apocynaceae & Leaves & Jaundice and liver diseases & [THH]; [83] \\
\hline 24 & $\begin{array}{l}\text { Carthamus oxycantha Bieb } \\
\text { [0024/ASAB/NUST] }\end{array}$ & Pohli & Asteraceae & seeds & Oil is used against itching & {$[\mathrm{THH}] ;[65]$} \\
\hline 25 & $\begin{array}{l}\text { Chenopodium album L. } \\
\text { [0025/ASAB/NUST] }\end{array}$ & Bathu & Chenopodiaceae & Whole plant & $\begin{array}{l}\text { Jaundice, urinary problems, } \\
\text { antidote against snake bite }\end{array}$ & [THH]; [36]; [65] \\
\hline 26 & $\begin{array}{l}\text { Cicer arietinum L. [0026/ } \\
\text { ASAB/NUST] }\end{array}$ & Chinnay & Papilionaceae & seeds & High blood sugar & {$[82]$} \\
\hline 27 & $\begin{array}{l}\text { Cichorium intybus L. [0027/ } \\
\text { ASAB/NUST] }\end{array}$ & Kasni & Asteraceae & Roots, whole plant & $\begin{array}{l}\text { High blood sugar, jaundice } \\
\text { and liver diseases }\end{array}$ & $\begin{array}{l}\text { [THH]; [66]; [82]; } \\
{[83]}\end{array}$ \\
\hline 28 & $\begin{array}{l}\text { Citrullus colocynthus (L.) } \\
\text { Schrad [0028/ASAB/NUST] }\end{array}$ & Tumma & Cucurbitaceae & Fruit & $\begin{array}{l}\text { Abdominal diseases, } \\
\text { constipation }\end{array}$ & {$[\mathrm{THH}] ;[67]$} \\
\hline 29 & $\begin{array}{l}\text { Convolvulus arvensis L. } \\
\text { [0029/ASAB/NUST] }\end{array}$ & Laili, erlai & Convolvulaceae & Aerial parts & $\begin{array}{l}\text { Abdominal worms, used as } \\
\text { deodorant, skin disorders }\end{array}$ & $\begin{array}{l}\text { [THH]; [36]; [65]; } \\
{[67]}\end{array}$ \\
\hline 30 & $\begin{array}{l}\text { Cucumis sativus L. [0030/ } \\
\text { ASAB/NUST] }\end{array}$ & Kheera & Cucurbitaceae & Fruit & Jaundice and liver diseases & [THH]; [83] \\
\hline 31 & $\begin{array}{l}\text { Cynodon dactylon L. [0031/ } \\
\text { ASAB/NUST] }\end{array}$ & Khabal ghas & Poaceae & Stem, leaves & Dysentery with fever & {$[65]$} \\
\hline 32 & $\begin{array}{l}\text { Cyperus rotundas L. [0032/ } \\
\text { ASAB/NUST] }\end{array}$ & Deela & Cyperaceae & Whole plant & $\begin{array}{l}\text { Indigestion, vomiting, } \\
\text { diarrhea and vomiting, fever }\end{array}$ & {$[65]$} \\
\hline 33 & $\begin{array}{l}\text { Dalbergia sissoo Roxb. } \\
\text { [0033/ASAB/NUST] }\end{array}$ & Tali & Papilionaceae & Leaves & Dandruff & {$[67]$} \\
\hline 34 & $\begin{array}{l}\text { Dodonaea viscosa (L.) Jacq. } \\
\text { [0034/ASAB/NUST] }\end{array}$ & Sanatha & Sapindaceae & Leaves & High blood sugar & {$[82]$} \\
\hline 35 & $\begin{array}{l}\text { Eucalyptus cammaldulensis } \\
\text { Dehn [0035/ASAB/NUST] }\end{array}$ & Sufaida, Lachi & Myrtaceae & Leaves & Flu & {$[\mathrm{THH}] ;[67]$} \\
\hline 36 & $\begin{array}{l}\text { Euphorbia helioscopia L. } \\
\text { [0036/ASAB/NUST] }\end{array}$ & Chattri dodak & Euphorbiaceae & Leaves, root, latex & $\begin{array}{l}\text { Constipation, increases milk } \\
\text { supply }\end{array}$ & {$[\mathrm{THH}] ;[65]$} \\
\hline 37 & $\begin{array}{l}\text { Euphorbia royleana Boiss. } \\
\text { [0037/ASAB/NUST] }\end{array}$ & Danda thor & Euphorbiaceae & branch & Ear pain & {$[67]$} \\
\hline 38 & $\begin{array}{l}\text { Fagonia indica Burm. } \\
\text { F. [0038/ASAB/NUST] }\end{array}$ & Dhamian & Zygophyllaceae & $\begin{array}{l}\text { Leaves and } \\
\text { branches }\end{array}$ & $\begin{array}{l}\text { Gas trouble, skin problems, } \\
\text { high blood sugar }\end{array}$ & {$[\mathrm{THH}] ;[67] ;[82]$} \\
\hline 39 & $\begin{array}{l}\text { Ficus bengalensis L. } \\
\text { [0039/ASAB/NUST] }\end{array}$ & Bohr & Moraceae & $\begin{array}{l}\text { Leaves and } \\
\text { branches latex }\end{array}$ & High blood sugar & {$[\mathrm{THH}] ;[82]$} \\
\hline 40 & $\begin{array}{l}\text { Foeniculum vulgare Mill. } \\
\text { [0040/ASAB/NUST] }\end{array}$ & Soonf & Apiaceae & inflorescence & $\begin{array}{l}\text { Eye-cataract, stomach } \\
\text { disorders, indigestion }\end{array}$ & {$[\mathrm{THH}] ;[67]$} \\
\hline 41 & $\begin{array}{l}\text { Fumaria indica (Husskn.) } \\
\text { H.N. Pugsley [0041/ASAB/ } \\
\text { NUST] }\end{array}$ & Shahtra papra & Fumariaceae & Whole plant & $\begin{array}{l}\text { Diarrhea, also used as blood } \\
\text { purifier }\end{array}$ & {$[\mathrm{THH}] ;[36]$} \\
\hline 42 & $\begin{array}{l}\text { Hordeum vulgare L. [0042/ } \\
\text { ASAB/NUST] }\end{array}$ & Jo & Poaceae & seeds & $\begin{array}{l}\text { Kidney pain, high blood } \\
\text { sugar; jaundice }\end{array}$ & [66]; [67]; [82]; [83] \\
\hline 43 & $\begin{array}{l}\text { Ipomoea pentaphylla (L.) } \\
\text { Jacq. [0043/ASAB/NUST] }\end{array}$ & Kaan Kati & Convolvulaceae & Seeds & $\begin{array}{l}\text { Jaundice, intestinal pain and } \\
\text { worms }\end{array}$ & {$[\mathrm{THH}] ;[36]$} \\
\hline 44 & $\begin{array}{l}\text { Justacia adhatoda L. [0044/ } \\
\text { ASAB/NUST] }\end{array}$ & Bahker & Acanthaceae & roots & Jaundice and liver diseases & {$[\mathrm{THH}] ;[83]$} \\
\hline 45 & $\begin{array}{l}\text { Kickxia ramosissima (Wall) } \\
\text { Janchen [0045/ASAB/NUST] }\end{array}$ & Khunger booti & Scrophulariaceae & Whole plant & High blood sugar & {$[82]$} \\
\hline 46 & $\begin{array}{l}\text { Lactuca serriola L. [0046/ } \\
\text { ASAB/NUST] }\end{array}$ & Kahu & Asteraceae & Whole plant & $\begin{array}{l}\text { Stomach ache, cough, and } \\
\text { asthma. }\end{array}$ & {$[\mathrm{THH}] ;[65]$} \\
\hline 47 & $\begin{array}{l}\text { Malva parviflora Wall. [0047/ } \\
\text { ASAB/NUST] }\end{array}$ & Sonchal & Malvaceae & Whole plant & Cough, flue and fever & {$[\mathrm{THH}] ;[36]$} \\
\hline 48 & $\begin{array}{l}\text { Melia azedarach L. [0048/ } \\
\text { ASAB/NUST] }\end{array}$ & $\begin{array}{l}\text { Dharek, bakain, } \\
\text { Herak }\end{array}$ & Meliaceae & Leaves, fruits & $\begin{array}{l}\text { Piles, foot itching, high blood } \\
\text { sugar, emotional disturbance, } \\
\text { blood pressure }\end{array}$ & $\begin{array}{l}\text { [THH]; [67]; [68]; } \\
{[82]}\end{array}$ \\
\hline
\end{tabular}


Table 3 Medicinal Flora of Pothwar Plateau (Northern Punjab) (Continued)

\begin{tabular}{|c|c|c|c|c|c|c|}
\hline 49 & $\begin{array}{l}\text { Momordica charantia L. } \\
\text { [0049/ASAB/NUST] }\end{array}$ & Karella & Cucurbitaceae & fruits & High blood sugar & {$[\mathrm{THH}] ;[82]$} \\
\hline 50 & $\begin{array}{l}\text { Morus alba L. [0050/ } \\
\text { ASAB/NUST] }\end{array}$ & $\begin{array}{l}\text { Shehtoot, Chitta } \\
\text { toot }\end{array}$ & Moraceae & Leaves, fruits & $\begin{array}{l}\text { Cough, sore throat, jaundice } \\
\text { and liver diseases }\end{array}$ & $\begin{array}{l}\text { [THH]; [36]; [67]; } \\
{[83]}\end{array}$ \\
\hline 51 & $\begin{array}{l}\text { Morus nigra L. [0051/ } \\
\text { ASAB/NUST] }\end{array}$ & Kalla toot & Moraceae & Fruit & $\begin{array}{l}\text { Cough, sore throat, Jaundice } \\
\text { and liver diseases }\end{array}$ & {$[\mathrm{THH}] ;[66] ;[83]$} \\
\hline 52 & $\begin{array}{l}\text { Myrsine africana L. } \\
\text { [0052/ASAB/NUST] }\end{array}$ & Khukan & Myrsinaceae & Leaves & Jaundice and liver diseases & {$[\mathrm{THH}] ;[83]$} \\
\hline 53 & $\begin{array}{l}\text { Ocimum album L. [0053/ } \\
\text { ASAB/NUST] }\end{array}$ & Chitti Tulsi & Lamiaceae & Leaves & High blood sugar & {$[36]$} \\
\hline 54 & $\begin{array}{l}\text { Ocimum basilicum L. } \\
\text { [0054/ASAB/NUST] }\end{array}$ & Niazbo & Lamiaceae & Leaves & Mouth sores & {$[\mathrm{THH}] ;[67]$} \\
\hline 55 & $\begin{array}{l}\text { Ocimum sanctum L. } \\
\text { [0055/ASAB/NUST] }\end{array}$ & Tulsi & Lamiaceae & Leaves & High blood sugar & {$[82]$} \\
\hline 57 & $\begin{array}{l}\text { Parthenium hysterophorus } \\
\text { L. [0057/ASAB/NUST] }\end{array}$ & Chatak chandni & Asteraceae & Whole plant & Severe diarrhea. & {$[36]$} \\
\hline 58 & $\begin{array}{l}\text { Peganum heramala } \mathrm{L} . \\
\text { [0058/ASAB/NUST] }\end{array}$ & Hermal & Zygophyllaceae & $\begin{array}{l}\text { Seeds and whole } \\
\text { plant }\end{array}$ & $\begin{array}{l}\text { Abdominal pain, also Smoke } \\
\text { has insecticidal properties }\end{array}$ & {$[\mathrm{THH}] ;[67]$} \\
\hline 59 & $\begin{array}{l}\text { Phyllanthus emblica L. } \\
\text { [0059/ASAB/NUST] }\end{array}$ & Aamla & Euphorbiaceae & fruits & Jaundice and liver diseases & {$[\mathrm{THH}] ;[83]$} \\
\hline 60 & $\begin{array}{l}\text { Plantago lanceolatum L. } \\
\text { [0060/ASAB/NUST] }\end{array}$ & Ispaghol & Plantaginaceae & Seed husk & $\begin{array}{l}\text { Gas trouble, indigestion, } \\
\text { stomach problems }\end{array}$ & {$[\mathrm{THH}] ;[67]$} \\
\hline 61 & $\begin{array}{l}\text { Plantago ovata Forssk. } \\
\text { [0061/ASAB/NUST] }\end{array}$ & Bhatti & Plantaginaceae & Fruit, Seed husk & Jaundice and liver diseases & {$[\mathrm{THH}] ;[66] ;[83]$} \\
\hline 62 & $\begin{array}{l}\text { Pongamia pinnata }(\mathrm{L} .) \\
\text { Merril }[0062 / \mathrm{ASAB} / \mathrm{NUST}]\end{array}$ & Sukh chain & Papilionaceae & Leaves, seeds, root & $\begin{array}{l}\text { Skin problems and } \\
\text { stomachache }\end{array}$ & {$[65]$} \\
\hline 64 & $\begin{array}{l}\text { Psidium guajava L. [0064/ } \\
\text { ASAB/NUST] }\end{array}$ & Amrood & Myrtaceae & leaves & $\begin{array}{l}\text { High blood pressure, high } \\
\text { blood sugar, constipation }\end{array}$ & {$[\mathrm{THH}] ;[67] ;[82]$} \\
\hline 65 & $\begin{array}{l}\text { Punica granatum L. [0065/ } \\
\text { ASAB/NUST] }\end{array}$ & Anar & Punicaceae & Fruit & Diarrhea, anemia & {$[\mathrm{THH}] ;[67]$} \\
\hline 66 & $\begin{array}{l}\text { Raphanus sativus L. [0066/ } \\
\text { ASAB/NUST] }\end{array}$ & Mooli & Brassicaceae & Root & Jaundice and liver diseases & {$[\mathrm{THH}] ;[83]$} \\
\hline 67 & $\begin{array}{l}\text { Rhazya stricta Decne. } \\
\text { [0067/ASAB/NUST] }\end{array}$ & $\begin{array}{l}\text { Vena, venra, } \\
\text { Verian }\end{array}$ & Apocynaceae & $\begin{array}{l}\text { Leaves and } \\
\text { branches }\end{array}$ & Tooth ache, acne & {$[67]$} \\
\hline 68 & $\begin{array}{l}\text { Ricinus communis L. } \\
\text { [0068/ASAB/NUST] }\end{array}$ & Arind & Euphorbiaceae & Leaves, oil & $\begin{array}{l}\text { Wound healing, constipation, } \\
\text { joints swelling and pain. }\end{array}$ & {$[\mathrm{THH}] ;[36] ;[67]$} \\
\hline 69 & $\begin{array}{l}\text { Rosa indica L. [0069/ } \\
\text { ASAB/NUST] }\end{array}$ & Gulab & Rosaceae & petals & $\begin{array}{l}\text { Eye burning, constipation, } \\
\text { abdominal pain. }\end{array}$ & {$[\mathrm{THH}]$} \\
\hline 70 & $\begin{array}{l}\text { Rumex hastatus D. Don, } \\
\text { Prodr. [0070/ASAB/NUST] }\end{array}$ & Khatimal & Polygonaceae & Leaves & Jaundice and liver diseases & {$[\mathrm{THH}] ;[83]$} \\
\hline 71 & $\begin{array}{l}\text { Silybum marianum L. } \\
\text { Gaertn [0071/ASAB/NUST] }\end{array}$ & $\begin{array}{l}\text { Ount Katara, } \\
\text { kandiali }\end{array}$ & Asteraceae & Leaves, seeds & Liver diseases, horse bite & {$[83] ;[84]$} \\
\hline 72 & $\begin{array}{l}\text { Sisymbrium irio L. [0072/ } \\
\text { ASAB/NUST] }\end{array}$ & $\begin{array}{l}\text { Khoob Kalan, } \\
\text { Jangli sarson }\end{array}$ & Brassicaceae & Leaves, seeds & $\begin{array}{l}\text { Throat, chest infection and } \\
\text { swelling }\end{array}$ & {$[\mathrm{THH}] ;[36] ;[65]$} \\
\hline 73 & $\begin{array}{l}\text { Solanum nigrum L. [0073/ } \\
\text { ASAB/NUST] }\end{array}$ & Kachmach & Solanaceae & $\begin{array}{l}\text { Leaves and } \\
\text { branches }\end{array}$ & $\begin{array}{l}\text { Abdominal pain, } \\
\text { stomachache, high blood } \\
\text { sugar, burnt skin and wounds }\end{array}$ & $\begin{array}{l}\text { [THH]; [37]; [67]; } \\
{[82]}\end{array}$ \\
\hline 74 & $\begin{array}{l}\text { Solanum surratense Burm. } \\
\text { F. [0074/ASAB/NUST] }\end{array}$ & Kandiari/Muhakri & Solanaceae & Fruits, flowers & $\begin{array}{l}\text { Abdomen pain, gas trouble, } \\
\text { chronic coughs and pain }\end{array}$ & {$[\mathrm{THH}] ;[36] ;[67]$} \\
\hline
\end{tabular}


Table 3 Medicinal Flora of Pothwar Plateau (Northern Punjab) (Continued)

\begin{tabular}{|c|c|c|c|c|c|c|}
\hline 75 & $\begin{array}{l}\text { Sonchus arvensis L. [0075/ } \\
\text { ASAB/NUST] }\end{array}$ & Dodak & Asterceae & Whole plant & $\begin{array}{l}\text { Jaundice, cough, chest } \\
\text { stiffness, asthma }\end{array}$ & {$[65]$} \\
\hline 76 & $\begin{array}{l}\text { Syzygium cumini (L.) Skeets. } \\
\text { [0076/ASAB/NUST] }\end{array}$ & Jaman & Myrtaceae & Seeds & High blood sugar & {$[\mathrm{THH}] ;[67]$} \\
\hline 77 & $\begin{array}{l}\text { Tagetes petala L. [0077/ } \\
\text { ASAB/NUST] }\end{array}$ & Satbarga & Asteraceae & Leaves & Ear pain & {$[67]$} \\
\hline 78 & $\begin{array}{l}\text { Tamarindus indica L. [0078/ } \\
\text { ASAB/NUST] }\end{array}$ & Imli & Caesalpinaceae & Fruit, Roots & Jaundice and liver diseases & {$[\mathrm{THH}] ;[83]$} \\
\hline 79 & $\begin{array}{l}\text { Taraxacum officinale Weber } \\
\text { [0079/ASAB/NUST] }\end{array}$ & Doddak, Hand & Asteraceae & Leaves, Rhizome & High blood sugar, jaundice & {$[\mathrm{THH}] ;[82] ;[83]$} \\
\hline 80 & $\begin{array}{l}\text { Trachyspermum copticum L. } \\
\text { [0080/ASAB/NUST] }\end{array}$ & Ajwain & Apiaceae & Seeds & Gas trouble, stomach upset & {$[\mathrm{THH}] ;[67]$} \\
\hline 81 & $\begin{array}{l}\text { Tribulus terristris L. [0081/ } \\
\text { ASAB/NUST] }\end{array}$ & Bhakra & Zygophyllaceae & $\begin{array}{l}\text { Whole plant, } \\
\text { leaves }\end{array}$ & $\begin{array}{l}\text { Joint, muscle pain, urinary } \\
\text { disorders, impotency }\end{array}$ & {$[\mathrm{THH}] ;[36] ;[83]$} \\
\hline 82 & $\begin{array}{l}\text { Trigonella foenum-graecum } \\
\text { L. [0082/ASAB/NUST] }\end{array}$ & Methri & Papilionaceae & seeds & High blood sugar & {$[\mathrm{THH}] ;[82]$} \\
\hline 83 & $\begin{array}{l}\text { Tylophora hirsuta L. [0083/ } \\
\text { ASAB/NUST] }\end{array}$ & Glow & Asclepiadaceae & branches & High blood sugar & {$[82]$} \\
\hline 84 & $\begin{array}{l}\text { Vigna mungo (Burm. f.) } \\
\text { Walp. [0084/ASAB/NUST] }\end{array}$ & Mung & Papilionaceae & seeds & High blood sugar & {$[82]$} \\
\hline 85 & $\begin{array}{l}\text { Withania somnifera (L.) } \\
\text { Dunal [0085/ASAB/NUST] }\end{array}$ & Aksun, asgand & Solanaceae & leaves & $\begin{array}{l}\text { Cure joint, muscle pain, } \\
\text { uterine diseases, used as } \\
\text { sexual stimulant. }\end{array}$ & {$[\mathrm{THH}] ;[36] ;[67]$} \\
\hline 86 & $\begin{array}{l}\text { Zizyphus nummularia (Burm. } \\
\text { F) Wight and Arn. [0086/ } \\
\text { ASAB/NUST] }\end{array}$ & Beri & Rhamnaceae & Leaves, fruits & $\begin{array}{l}\text { Hair roughness, high blood } \\
\text { sugar, wound healing }\end{array}$ & $\begin{array}{l}\text { [THH]; [36]; [67]; } \\
{[82]}\end{array}$ \\
\hline Sr. No. & $\begin{array}{l}\text { Plant Name [voucher } \\
\text { specimen \#] }\end{array}$ & Vernacular name & Family & Plant part used & Disease Cure & References \\
\hline 1 & $\begin{array}{l}\text { Abutilon indicum G. Don } \\
{[0001 / A S A B / N U S T]}\end{array}$ & Kanghi & Malvaceae & Whole plant & $\begin{array}{l}\text { Diarrhea, sexually transmitted } \\
\text { diseases, burning with } \\
\text { urination }\end{array}$ & $\begin{array}{l}\text { Traditional Health } \\
\text { Healers [THH]; [41] }\end{array}$ \\
\hline 2 & $\begin{array}{l}\text { Acacia modesta Wall. } \\
{[0002 / A S A B / N U S T]}\end{array}$ & Phulahi & Mimosaceae & Bark of tree & $\begin{array}{l}\text { gas trouble and abdominal } \\
\text { diseases }\end{array}$ & {$[\mathrm{THH}] ;[43]$} \\
\hline 3 & $\begin{array}{l}\text { Acacia nilotica (L.) Delile. } \\
\text { [0003/ASAB/NUST] }\end{array}$ & Kiker & Mimosaceae & $\begin{array}{l}\text { Bark, leaves and } \\
\text { branches }\end{array}$ & $\begin{array}{l}\text { Mouth sores, gum pain and } \\
\text { toothache, eye sores, sexual } \\
\text { disability, diarrhea, asthma }\end{array}$ & {$[\mathrm{THH}] ;[43] ;[44]$} \\
\hline 4 & $\begin{array}{l}\text { Achyranthus aspera L. } \\
\text { [0004/ASAB/NUST] }\end{array}$ & Puth kanda & Amaranthaceae & Whole plant & $\begin{array}{l}\text { Excessive menstruation, piles, } \\
\text { abdominal pain, toothache, } \\
\text { severe diarrhea. }\end{array}$ & {$[\mathrm{THH}] ;[41]$} \\
\hline 5 & $\begin{array}{l}\text { Adhatoda vasica Nees } \\
\text { [0005/ASAB/NUST] }\end{array}$ & Bekkar & Acanthaceae & Leaves & High blood sugar & {$[\mathrm{THH}] ;[59]$} \\
\hline 6 & $\begin{array}{l}\text { Adiantum capillus veneris L. } \\
\text { [0006/ASAB/NUST] }\end{array}$ & Sarhaj & Adiantaceae & Leaves & Jaundice and liver diseases & {$[\mathrm{THH}] ;[61]$} \\
\hline 7 & $\begin{array}{l}\text { Albizzia lebbek (L.) Benth } \\
\text { [0007/ASAB/NUST] }\end{array}$ & Shrin & Mimosaceae & Leaves & Eye problems & {$[\mathrm{THH}] ;[43]$} \\
\hline 8 & $\begin{array}{l}\text { Allium cepa L. [0008/ } \\
\text { ASAB/NUST] }\end{array}$ & Piaz & Liliaceae & bulb & $\begin{array}{l}\text { High blood pressure, high } \\
\text { blood sugar }\end{array}$ & {$[\mathrm{THH}] ;[59]$} \\
\hline 9 & $\begin{array}{l}\text { Allium sativum L. [0009/ } \\
\text { ASAB/NUST] }\end{array}$ & Thoom & Liliaceae & bulb & $\begin{array}{l}\text { Ear pain, hypertension, high } \\
\text { blood sugar }\end{array}$ & {$[\mathrm{THH}] ;[43] ;[59]$} \\
\hline 10 & $\begin{array}{l}\text { Aloe vera L. [0010/ASAB/ } \\
\text { NUST] }\end{array}$ & Knwar gandal & Liliaceae & Leaf sap, stem & $\begin{array}{l}\text { Abdominal pains, } \\
\text { constipation, skin diseases, } \\
\text { high blood sugar }\end{array}$ & {$[\mathrm{THH}] ;[43] ;[59]$} \\
\hline 11 & $\begin{array}{l}\text { Amaranthus viridis L. } \\
\text { [0011/ASAB/NUST] }\end{array}$ & Chaulai & Amaranthaceae & Leaves & $\begin{array}{l}\text { Menstrual disturbance, } \\
\text { constipation }\end{array}$ & {$[\mathrm{THH}] ;[41] ;[57]$} \\
\hline 12 & $\begin{array}{l}\text { Anethum graveolense L. } \\
\text { [0012/ASAB/NUST] }\end{array}$ & Soye & Apiaceae & seeds & Abdominal pain & [THH]; [43] \\
\hline
\end{tabular}


Table 3 Medicinal Flora of Pothwar Plateau (Northern Punjab) (Continued)

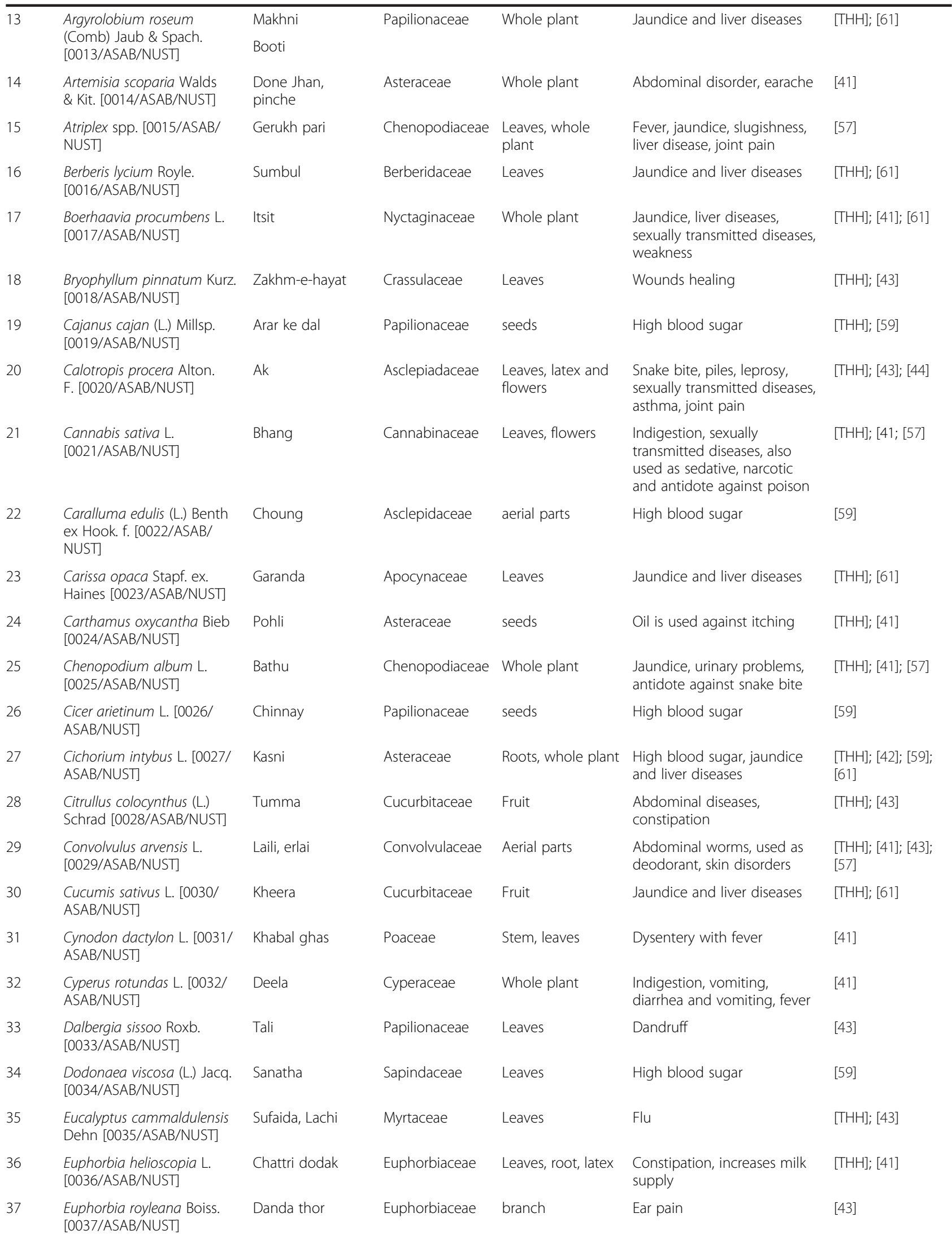


Table 3 Medicinal Flora of Pothwar Plateau (Northern Punjab) (Continued)

\begin{tabular}{|c|c|c|c|c|c|c|}
\hline 38 & $\begin{array}{l}\text { Fagonia indica Burm. F. } \\
\text { [0038/ASAB/NUST] }\end{array}$ & Dhamian & Zygophyllaceae & $\begin{array}{l}\text { Leaves and } \\
\text { branches }\end{array}$ & $\begin{array}{l}\text { Gas trouble, skin problems, } \\
\text { high blood sugar }\end{array}$ & {$[\mathrm{THH}] ;[43] ;[59]$} \\
\hline 39 & $\begin{array}{l}\text { Ficus bengalensis L. [0039/ } \\
\text { ASAB/NUST] }\end{array}$ & Bohr & Moraceae & $\begin{array}{l}\text { Leaves and } \\
\text { branches latex }\end{array}$ & High blood sugar & [THH]; [59] \\
\hline 40 & $\begin{array}{l}\text { Foeniculum vulgare Mill. } \\
\text { [0040/ASAB/NUST] }\end{array}$ & Soonf & Apiaceae & inflorescence & $\begin{array}{l}\text { Eye-cataract, stomach } \\
\text { disorders, indigestion }\end{array}$ & [THH]; [43] \\
\hline 41 & $\begin{array}{l}\text { Fumaria indica (Husskn.) } \\
\text { H.N. Pugsley [0041/ASAB/ } \\
\text { NUST] }\end{array}$ & Shahtra papra & Fumariaceae & Whole plant & $\begin{array}{l}\text { Diarrhea, also used as blood } \\
\text { purifier }\end{array}$ & {$[\mathrm{THH}] ;[57]$} \\
\hline 42 & $\begin{array}{l}\text { Hordeum vulgare L. [0042/ } \\
\text { ASAB/NUST] }\end{array}$ & Jo & Poaceae & seeds & $\begin{array}{l}\text { Kidney pain, high blood } \\
\text { sugar; jaundice }\end{array}$ & [42]; [43]; [59]; [61] \\
\hline 43 & $\begin{array}{l}\text { Ipomoea pentaphylla (L.) } \\
\text { Jacq. [0043/ASAB/NUST] }\end{array}$ & Kaan Kati & Convolvulaceae & Seeds & $\begin{array}{l}\text { Jaundice, intestinal pain and } \\
\text { worms }\end{array}$ & {$[\mathrm{THH}] ;[57]$} \\
\hline 44 & $\begin{array}{l}\text { Justacia adhatoda L. [0044/ } \\
\text { ASAB/NUST] }\end{array}$ & Bahker & Acanthaceae & roots & Jaundice and liver diseases & {$[\mathrm{THH}] ;[61]$} \\
\hline 45 & $\begin{array}{l}\text { Kickxia ramosissima (Wall) } \\
\text { Janchen [0045/ASAB/NUST] }\end{array}$ & Khunger booti & Scrophulariaceae & Whole plant & High blood sugar & {$[59]$} \\
\hline 46 & $\begin{array}{l}\text { Lactuca serriola L. [0046/ } \\
\text { ASAB/NUST] }\end{array}$ & Kahu & Asteraceae & Whole plant & $\begin{array}{l}\text { Stomach ache, cough, and } \\
\text { asthma. }\end{array}$ & [THH]; [41] \\
\hline 47 & $\begin{array}{l}\text { Malva parviflora Wall. } \\
\text { [0047/ASAB/NUST] }\end{array}$ & Sonchal & Malvaceae & Whole plant & Cough, flue and fever & {$[\mathrm{THH}] ;[57]$} \\
\hline 48 & $\begin{array}{l}\text { Melia azedarach L. } \\
\text { [0048/ASAB/NUST] }\end{array}$ & $\begin{array}{l}\text { Dharek, bakain, } \\
\text { Herak }\end{array}$ & Meliaceae & Leaves, fruits & $\begin{array}{l}\text { Piles, foot itching, high blood } \\
\text { sugar, emotional disturbance, } \\
\text { blood pressure }\end{array}$ & $\begin{array}{l}\text { [THH]; [43]; [44]; } \\
{[59]}\end{array}$ \\
\hline 49 & $\begin{array}{l}\text { Momordica charantia L. } \\
\text { [0049/ASAB/NUST] }\end{array}$ & Karella & Cucurbitaceae & fruits & High blood sugar & {$[\mathrm{THH}] ;[59]$} \\
\hline 50 & $\begin{array}{l}\text { Morus alba L. [0050/ } \\
\text { ASAB/NUST] }\end{array}$ & $\begin{array}{l}\text { Shehtoot, Chitta } \\
\text { toot }\end{array}$ & Moraceae & Leaves, fruits & $\begin{array}{l}\text { Cough, sore throat, jaundice } \\
\text { and liver diseases }\end{array}$ & $\begin{array}{l}{[\mathrm{THH}] ;[43] ;[57] ;} \\
{[61]}\end{array}$ \\
\hline 51 & $\begin{array}{l}\text { Morus nigra L. [0051/ } \\
\text { ASAB/NUST] }\end{array}$ & Kalla toot & Moraceae & Fruit & $\begin{array}{l}\text { Cough, sore throat, Jaundice } \\
\text { and liver diseases }\end{array}$ & {$[\mathrm{THH}] ;[42] ;[61]$} \\
\hline 52 & $\begin{array}{l}\text { Myrsine africana L. [0052/ } \\
\text { ASAB/NUST] }\end{array}$ & Khukan & Myrsinaceae & Leaves & Jaundice and liver diseases & {$[\mathrm{THH}] ;[61]$} \\
\hline 53 & $\begin{array}{l}\text { Ocimum album L. [0053/ } \\
\text { ASAB/NUST] }\end{array}$ & Chitti Tulsi & Lamiaceae & Leaves & High blood sugar & {$[57]$} \\
\hline 54 & $\begin{array}{l}\text { Ocimum basilicum L. } \\
\text { [0054/ASAB/NUST] }\end{array}$ & Niazbo & Lamiaceae & Leaves & Mouth sores & {$[\mathrm{THH}] ;[43]$} \\
\hline 55 & $\begin{array}{l}\text { Ocimum sanctum L. } \\
\text { [0055/ASAB/NUST] }\end{array}$ & Tulsi & Lamiaceae & Leaves & High blood sugar & {$[59]$} \\
\hline 56 & $\begin{array}{l}\text { Oxalis corniculata L. } \\
\text { [0056/ASAB/NUST] }\end{array}$ & $\begin{array}{l}\text { Gandora, khati } \\
\text { booti }\end{array}$ & Oxalidaceae & Leaves, seeds & $\begin{array}{l}\text { Jaundice, liver diseases, } \\
\text { stomach disorder }\end{array}$ & {$[43] ;[57] ;[61]$} \\
\hline 57 & $\begin{array}{l}\text { Parthenium hysterophorus } \\
\text { L. [0057/ASAB/NUST] }\end{array}$ & Chatak chandni & Asteraceae & Whole plant & Severe diarrhea. & {$[57]$} \\
\hline 58 & $\begin{array}{l}\text { Peganum heramala L. } \\
\text { [0058/ASAB/NUST] }\end{array}$ & Hermal & Zygophyllaceae & $\begin{array}{l}\text { Seeds and whole } \\
\text { plant }\end{array}$ & $\begin{array}{l}\text { Abdominal pain, also Smoke } \\
\text { has insecticidal properties }\end{array}$ & {$[\mathrm{THH}] ;[43]$} \\
\hline 59 & $\begin{array}{l}\text { Phyllanthus emblica L. } \\
\text { [0059/ASAB/NUST] }\end{array}$ & Aamla & Euphorbiaceae & fruits & Jaundice and liver diseases & {$[\mathrm{THH}] ;[61]$} \\
\hline 60 & $\begin{array}{l}\text { Plantago lanceolatum L. } \\
\text { [0060/ASAB/NUST] }\end{array}$ & Ispaghol & Plantaginaceae & Seed husk & $\begin{array}{l}\text { Gas trouble, indigestion, } \\
\text { stomach problems }\end{array}$ & {$[\mathrm{THH}] ;[43]$} \\
\hline 61 & $\begin{array}{l}\text { Plantago ovata Forssk. } \\
\text { [0061/ASAB/NUST] }\end{array}$ & Bhatti & Plantaginaceae & Fruit, Seed husk & Jaundice and liver diseases & {$[\mathrm{THH}] ;[42] ;[61]$} \\
\hline 62 & $\begin{array}{l}\text { Pongamia pinnata }(\mathrm{L} .) \\
\text { Merril }[0062 / \mathrm{ASAB} / \mathrm{NUST}]\end{array}$ & Sukh chain & Papilionaceae & Leaves, seeds, root & $\begin{array}{l}\text { Skin problems and } \\
\text { stomachache }\end{array}$ & {$[41]$} \\
\hline 63 & $\begin{array}{l}\text { Praecitrullus fistulosus } \\
\text { (Stocks.) Pangalo. } \\
\text { [0063/ASAB/NUST] }\end{array}$ & Teenda & Cucurbitaceae & leaves & Blood pressure & {$[43]$} \\
\hline
\end{tabular}


Table 3 Medicinal Flora of Pothwar Plateau (Northern Punjab) (Continued)

\begin{tabular}{|c|c|c|c|c|c|c|}
\hline 64 & $\begin{array}{l}\text { Psidium guajava L. [0064/ } \\
\text { ASAB/NUST] }\end{array}$ & Amrood & Myrtaceae & leaves & $\begin{array}{l}\text { High blood pressure, high } \\
\text { blood sugar, constipation }\end{array}$ & {$[\mathrm{THH}] ;[43] ;[59]$} \\
\hline 65 & $\begin{array}{l}\text { Punica granatum L. [0065/ } \\
\text { ASAB/NUST] }\end{array}$ & Anar & Punicaceae & Fruit & Diarrhea, anemia & {$[\mathrm{THH}] ;[43]$} \\
\hline 66 & $\begin{array}{l}\text { Raphanus sativus L. [0066/ } \\
\text { ASAB/NUST] }\end{array}$ & Mooli & Brassicaceae & Root & Jaundice and liver diseases & {$[\mathrm{THH}] ;[61]$} \\
\hline 67 & $\begin{array}{l}\text { Rhazya stricta Decne. [0067/ } \\
\text { ASAB/NUST] }\end{array}$ & $\begin{array}{l}\text { Vena, venra, } \\
\text { Verian }\end{array}$ & Apocynaceae & $\begin{array}{l}\text { Leaves and } \\
\text { branches }\end{array}$ & Tooth ache, acne & {$[43]$} \\
\hline 68 & $\begin{array}{l}\text { Ricinus communis L. [0068/ } \\
\text { ASAB/NUST] }\end{array}$ & Arind & Euphorbiaceae & Leaves, oil & $\begin{array}{l}\text { Wound healing, constipation, } \\
\text { joints swelling and pain. }\end{array}$ & {$[\mathrm{THH}] ;[43] ;[57]$} \\
\hline 69 & $\begin{array}{l}\text { Rosa indica L. [0069/ } \\
\text { ASAB/NUST] }\end{array}$ & Gulab & Rosaceae & petals & $\begin{array}{l}\text { Eye burning, constipation, } \\
\text { abdominal pain. }\end{array}$ & {$[\mathrm{THH}]$} \\
\hline 70 & $\begin{array}{l}\text { Rumex hastatus D. Don, } \\
\text { Prodr. [0070/ASAB/NUST] }\end{array}$ & Khatimal & Polygonaceae & Leaves & Jaundice and liver diseases & {$[\mathrm{THH}] ;[61]$} \\
\hline 71 & $\begin{array}{l}\text { Silybum marianum L. } \\
\text { Gaertn [0071/ASAB/NUST] }\end{array}$ & $\begin{array}{l}\text { Ount Katara, } \\
\text { kandiali }\end{array}$ & Asteraceae & Leaves, seeds & $\begin{array}{l}\text { Liver diseases, stomach } \\
\text { diseases }\end{array}$ & {$[61] ;[62]$} \\
\hline 72 & $\begin{array}{l}\text { Sisymbrium irio L. [0072/ } \\
\text { ASAB/NUST] }\end{array}$ & $\begin{array}{l}\text { Khoob Kalan, } \\
\text { Jangli sarson }\end{array}$ & Brassicaceae & Leaves, seeds & $\begin{array}{l}\text { Throat, chest infection and } \\
\text { swelling }\end{array}$ & {$[\mathrm{THH}] ;[41] ;[57]$} \\
\hline 73 & $\begin{array}{l}\text { Solanum nigrum L. [0073/ } \\
\text { ASAB/NUST] }\end{array}$ & Kachmach & Solanaceae & $\begin{array}{l}\text { Leaves and } \\
\text { branches }\end{array}$ & $\begin{array}{l}\text { Abdominal pain, } \\
\text { stomachache, high blood } \\
\text { sugar, burnt skin and wounds }\end{array}$ & $\begin{array}{l}\text { [THH]; [43]; [59]; } \\
{[60]}\end{array}$ \\
\hline 74 & $\begin{array}{l}\text { Solanum surratense Burm. F. } \\
\text { [0074/ASAB/NUST] }\end{array}$ & Kandiari/Muhakri & Solanaceae & Fruits, flowers & $\begin{array}{l}\text { Abdomen pain, gas trouble, } \\
\text { chronic coughs and pain }\end{array}$ & {$[\mathrm{THH}] ;[43] ;[57] ;$} \\
\hline 75 & $\begin{array}{l}\text { Sonchus arvensis L. [0075/ } \\
\text { ASAB/NUST] }\end{array}$ & Dodak & Asterceae & Whole plant & $\begin{array}{l}\text { Jaundice, cough, chest } \\
\text { stiffness, asthma }\end{array}$ & {$[41]$} \\
\hline 76 & $\begin{array}{l}\text { Syzygium cumini (L.) Skeets. } \\
\text { [0076/ASAB/NUST] }\end{array}$ & Jaman & Myrtaceae & Seeds & High blood sugar & {$[\mathrm{THH}] ;[43]$} \\
\hline 77 & $\begin{array}{l}\text { Tagetes petala L. [0077/ } \\
\text { ASAB/NUST] }\end{array}$ & Satbarga & Asteraceae & Leaves & Ear pain & {$[43]$} \\
\hline 78 & $\begin{array}{l}\text { Tamarindus indica L. } \\
\text { [0078/ASAB/NUST] }\end{array}$ & Imli & Caesalpinaceae & Fruit, Roots & Jaundice and liver diseases & {$[\mathrm{THH}] ;[61]$} \\
\hline 79 & $\begin{array}{l}\text { Taraxacum officinale } \\
\text { Weber [0079/ASAB/NUST] }\end{array}$ & Doddak, Hand & Asteraceae & Leaves, Rhizome & High blood sugar, jaundice & {$[\mathrm{THH}] ;[59] ;[61]$} \\
\hline 80 & $\begin{array}{l}\text { Trachyspermum copticum L. } \\
\text { [0080/ASAB/NUST] }\end{array}$ & Ajwain & Apiaceae & Seeds & Gas trouble, stomach upset & {$[\mathrm{THH}] ;[43]$} \\
\hline 81 & $\begin{array}{l}\text { Tribulus terristris L. [0081/ } \\
\text { ASAB/NUST] }\end{array}$ & Bhakra & Zygophyllaceae & $\begin{array}{l}\text { Whole plant, } \\
\text { leaves }\end{array}$ & $\begin{array}{l}\text { Joint, muscle pain, urinary } \\
\text { disorders, impotency }\end{array}$ & {$[\mathrm{THH}] ;[57] ;[61]$} \\
\hline 82 & $\begin{array}{l}\text { Trigonella foenum-graecum } \\
\text { L. [0082/ASAB/NUST] }\end{array}$ & Methri & Papilionaceae & seeds & High blood sugar & {$[\mathrm{THH}] ;[59]$} \\
\hline 83 & $\begin{array}{l}\text { Tylophora hirsuta L. [0083/ } \\
\text { ASAB/NUST] }\end{array}$ & Glow & Asclepiadaceae & branches & High blood sugar & {$[59]$} \\
\hline 84 & $\begin{array}{l}\text { Vigna mungo (Burm. f.) } \\
\text { Walp. [0084/ASAB/NUST] }\end{array}$ & Mung & Papilionaceae & seeds & High blood sugar & {$[59]$} \\
\hline 85 & $\begin{array}{l}\text { Withania somnifera (L.) } \\
\text { Dunal [0085/ASAB/NUST] }\end{array}$ & Aksun, asgand & Solanaceae & leaves & $\begin{array}{l}\text { Cure joint, muscle pain, } \\
\text { uterine diseases, used as } \\
\text { sexual stimulant. }\end{array}$ & {$[\mathrm{THH}] ;[43] ;[57]$} \\
\hline 86 & $\begin{array}{l}\text { Zizyphus nummularia (Burm. } \\
\text { F) Wight and Arn. [0086/ } \\
\text { ASAB/NUST] }\end{array}$ & Beri & Rhamnaceae & Leaves, fruits & $\begin{array}{l}\text { Hair roughness, high blood } \\
\text { sugar, wound healing }\end{array}$ & $\begin{array}{l}\text { [THH]; [43]; [57]; } \\
{[59]}\end{array}$ \\
\hline
\end{tabular}

\section{Plant parts used}

Plants as whole and different parts are commonly used to cure different diseases in the study area. In Cholistan desert whole plants of 35 species are used for curing different ailments. Leaves of 17 plants are used to prepare different medicines. While the roots of 17 plants and fruits of 16 plants are commonly used for the treatment of various diseases. Seeds/nuts of 15 plants bark 14 and are being used to treat several ailments. Stems/twigs of 13 plants are commonly used, whereas gum/resin 13, 
latex 12, floral buds of 6 plants and thorns of 2 plants are used as medicine. Among all the plants studied in Pothwar Plateau leaves of most plants (39) are used in herbal remedies to cure different ailments while whole plants and seeds of 17 of these are mostly used medicinally by local inhabitants. Percentage of all plants parts used is depicted in Figure 2.

Twenty eight therapeutic plants are used in the form of decoction and paste (used externally). 25 plant species are used in the form powder and syrup of 12 plant species are prepared before their use as medicine. Infusion of 11 plant species, fresh juice of 8 plant species and tablets of 7 plant species are prepared and used as medicine. "Majoon" /electuary of 7 plant species, ash or salt of 5 plant species and mother tincture of 4 plants are used to cure different diseases in Cholistan Desert areas.

\section{Diagnosis and treatment of diseases}

Traditional healers commonly diagnose illness by visual inspection, interviewing patients for symptoms and the duration of the health problem. Symptoms related to variation in pulse rate, eye color, skin color, mouth infections, body temperature and condition of sores, are the basis for prescription of remedy. Internal disorders are usually cured by recommending the herbal preparations in the form of syrup, while external wounds and dermal infections are treated by applying and rubbing herbal preparations on the affected parts.

Ethno-pharmacologically prepared drugs are traditionally administered with either medium like water, cow/buffalo milk, goat milk, sheep milk, "lassi" and "arq" (distillate). These media are commonly advised according to the condition of the patient, age and nature of the disease. The main purpose of these liquids is for good absorption of medicine, to minimize the side effects (if any) of given remedy, and sometimes these are recommended to provide the nutritional support to the patient along with medicines.
During present study it has been observed that water is recommended for medicine preferably than followed by cow/buffalo milk, goat milk, sheep milk, "lassi" and "arq".

The comprehensive information on ethno-medicinal flora of Cholistan desert and Pothwar with regard to scientific names, local names, family, part used and diseases cure have been provided in Tables 2 and 3 respectively.

The survey of both areas revealed the variation in recommended dosages of medicinal plants among the traditional healers for treating the same disease. The traditional healers believed that the doses for liquid preparations are prescribed in terms of a full, half or one fourth of a cup, depending on the age, physical condition of the patient being treated, type of illness, diagnosis and severity of the disease. There is no standardized criterion for the dosage of herbal remedies. The quantities of preparation and prescription rates generally vary with the degree and duration of the ailment. The age group of the patient, type and level of disease further decide the rate of treatment. Lack of standard procedures and quality control is seen as a common problem of conventional medicines in the developing world [38].

During the recent past ethnobotanic research has been done tremendously to explore, use and conserve natural resources especially in search of novel crude drugs. Medicinal plants are a significant source of phytochemicals that are of great importance for the health of individuals and communities [38]. Geographically quite many ethnobotanic studies have been published in recent years in Pakistan [2]. Nevertheless some of them are mentioned here. During past 5 years most of the studies are done in mountain regions [39-43]. The studies are mainly focused on listing of ethnobotanic uses of plants. Some of the plants reported have also been proven scientifically to contain valuable medicinal properties [44-48]. Several ethnobotanic surveys done from Punjab province include Soan Valley [49], Thal desert [50], Khusab [51] and

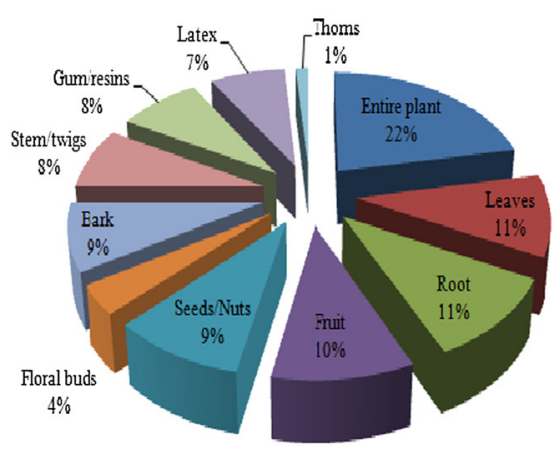

a

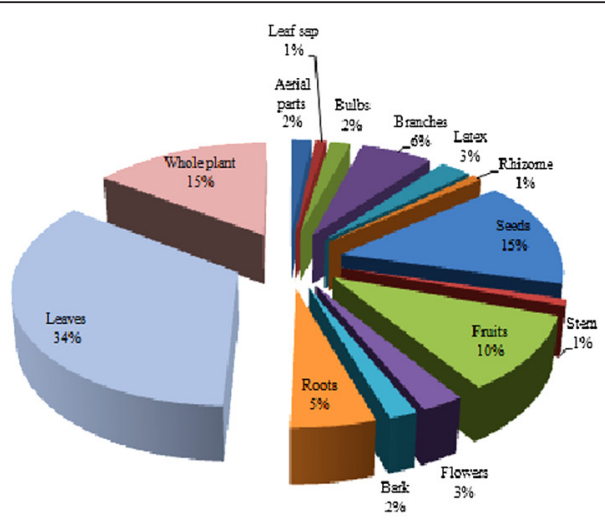

$\mathrm{b}$

Figure 2 Plant parts (\%) used as medicine against different diseases in a. Cholistan Desert and $\mathbf{b}$. Pothwar Plateau. 
Sialkot [52]. Specific studies done on Cholistan area include [53-59]. The present study also supports the usages reported by earlier studies cited in Tables 2 and 3.

\section{Conclusions and recommendations}

The reported number of medicinal plants (67 species) in the native folklore remedies seems very significant considering the vegetation cover of Cholistan as the area has very sparse vegetation. The prolonged and reoccurring drought phenomenon, environmental degradation, grazing pressure and wood cutting for fuel purpose are the notable factors worth consideration to assess the significance of medicinal plants of Cholistan.

It can be concluded from the present study that Cholistan desert and arid areas of Pothwar (86 Species) are rich in indigenous medicinal plant wealth. Data acquired on medicinal plants of Northern Punjab (Pothwar area) and Southern Punjab (Cholistan) bears only $10.5 \%$ similarity. The total number of population in the area also justifies the high number of plants species under medicinal use. The local people of both areas possess a wealth of traditional knowledge. Plants of semiarid area of Pothwar have comparatively been explored more scientifically. The present information provides basis for the recognition of this undocumented knowledge but will also help in conservation of such an important desert species of Cholistan. It also opens the way for further investigation in new dimensions for better healthcare of human being regarding various diseases. This study has highlighted that old indigenous knowledge of traditional medicine is still in use predominantly in Cholistan desert. Many folk remedies used in traditional medicine are used as a first line of health care. The trust of the respondents on traditional medicine regarding its efficacy and cost effectiveness establishes its preference over the modern allopathic drugs. But there is variation in use of these folk remedies in both areas. The faith of the Cholistani pastorals is more in the traditional medicines. As the folklore knowledge has been passed on generally verbally from generation to generation and not documented, it is gradually fading. So it is recommended that research and developmental efforts should be focused on these plants species to scientifically identify the plant potential and substantially improve the traditional herbal therapies of the rural people.

The observations emerged from the present study should be substantiated with pharmaco-chemical studies in order to evaluate their effectiveness. The study indicated that the area of study have plenty of medicinal plants to treat a wide range of human ailments. Present study revealed that the local people prefer folk medicine due to low cost and sometimes it is a part of their social life and culture. It develops proactive link between shortterm actions to long-term goals and offers analytical tools to the survival of human communities. Therefore, it is imperative to acquire and preserve this traditional system of plant utilization by proper documentation and identification of specimen. This work presents a review focusing on the main historical and current interactions between humans and the flora, the ecological implications and the role of the ethnobotany in plants conservation. The importance of ethnobiological studies for biodiversity conservation has increasingly been recognized. Sustainable harvesting of these plants is an essential component of this study to conserve natural sources. Thus there is a need to create awareness about the importance of these plants among local people and to provide them guidance and training in collection and processing to enhance the economic benefits from their indigenous flora.

\section{Competing interests}

The authors declare that they have no competing interests.

\section{Authors' contributions}

All authors contributed equally and approved the final version of the manuscript. MQH and SA supervised the whole work.

\section{Acknowledgements}

We acknowledge National University of Sciences and Technology (NUST), H-12 Islamabad, Pakistan and The Islamia University of Bahawalpur, Pakistan for funding. We are also obliged to Prof. Dr. Atta-ur-Rahman (FRS, NI, HI, Sl, $\mathrm{TI})$ for his generous support towards ASAB and CIDS collaboration.

\section{Author details}

'Medicinal Plant Research Laboratory, Department of Plant Biotechnology, Atta-ur-Rahman School of Applied Biosciences (ASAB), National University of Sciences and Technology (NUST), Islamabad, Pakistan. ${ }^{2}$ University College of Agriculture, University of Sargodha, Sargodha, Pakistan. ${ }^{3}$ Cholistan Institute of Desert Studies (CIDS), The Islamia University of Bahawalpur, Bahawalpur, Pakistan. ${ }^{4}$ Department of Biotechnology, American University of Ras Al Khaimah, PO Box 10021, Ras Al Khaimah, United Arab Emirates.

Received: 6 November 2013 Accepted: 31 March 2015

Published online: 30 April 2015

\section{References}

1. Ali SI, Qaiser M. Proceedings of Royal Society Edinburgh, vol. 89B. 1986. p. 89-101.

2. Shinwari MI, Shinwari MI. Botanical diversity in Pakistan, past, present and future. In: Proceedings of Seminar on World Environment Day: 2010. Lahore: Pakistan Engineering Congress; 2010. p. 85-104.

3. Ahmad S, Alam K, Wariss HM, Anjum S, Mukhtar M. Ethnobotanical studies of plant resources of Cholistan desert, Pakistan. Int J Sci Res. 2014;3:1782-8.

4. Shinwari ZK. Medicinal plants research in Pakistan. J Med Plants Res. 2010;4:161-76.

5. Ghayur MN, Kazim SF, Rasheed H, Khalid A, Jumani Ml, Choudhary Ml, et al. Identification of antiplatelet and acetylcholinesterase inhibitory constituents in betel nut. Zhong Xi Yi Jie He Xue Bao. 2011;9:619-25.

6. Ahmed T, Gilani AH. A comparative study of curcuminoids to measure their effect on inflammatory and apoptotic gene expression in an $A \beta$ plus ibotenic acid-infused rat model of Alzheimer's disease. Brain Res. 2011;11:1-18.

7. Khan S, Mehmood MH, Ali AN, Ahmed FS, Dar A, Gilani AH. Studies on anti-inflammatory and analgesic activities of betel nut in rodents. J Ethnopharmacol. 2011;135:654-61.

8. Mehmood MH, Aziz N, Ghayur MN, Gilani AH. Pharmacological basis for the medicinal use of Psyllium husk (Ispaghula) in constipation and diarrhea. Dig Dis Sci. 2011;56:1460-71.

9. Malik F, Hussain S, Mirza T, Hameed A, Ahmad S, Usmanghani K. Screening for antimicrobial activity of thirty three medicinal plants used in the traditional system of medicine in Pakistan. J Med Plants Res. 2011;5:3052-60. 
10. Hayat MQ, Khan MA, Ahmad M, Shaheen N, Yasmin G, Akhter S. Ethno taxonomical approach in the identification of useful medicinal flora of Tehsil Pindigheb (District Attock) Pakistan. Ethnobot Res Applic. 2008;6:35-062.

11. Wariss HM, Ahmad S, Anjum S, Alam K. Ethnobotanical studies of dicotyledonous plants of Lal Suhanra National Park, Bahawalpur, Pakistan. Int J Sci Res. 2014;3:2452-60.

12. Ahmad M, Khan MA, Zafar M, Sultana S. Treatment of common ailments by plant based remedies among the people of District Attock (Punjab) of Northern Pakistan. Afr J Trad Compliment Altern Med. 2007;4:112-20.

13. Ali I, Chaudhary MS, Farooq U. Camel rearing in Cholistan Desert of Pakistan. Pak Vet J. 2009;29:85-92

14. Ahmad TF, Akbar G, Tahir MB, Ahmad I. Developing Cholistan desert - a perspective. Prog Farming. 1992;12:35-40.

15. Auj ANZ. Legacy of Cholistan. Multan, Pakistan: Caravan Book Centre; 1995

16. Ahmad F. Eco-regeneration and runoff collection in Cholistan. UNEP Desert C Bull. 1999;35:50-4.

17. Ahmad F. Eco-regeneration and runoff collection in Cholistan. In Proceedings of an International Conference on Desertification and Soil Degradation: 11-15 November 1999. Moscow; 1999. p. 337-43.

18. Arshad M, Ashraf MY, Ahmad M, Zaman F. Morpho-genetic variability potential of Cenchrus ciliaris L., from Cholistan desert, Pakistan. Pak J Bot. 2007;39:1481-8.

19. Ahmad F. Historical and archaeological perspectives of soil degradation in Cholistan. J Geophys Res. 2005;8:31-5.

20. Akbar G, Khan TN, Arshad M. Cholistan desert. Pak Rangel. 1996;18:124-8

21. Chaudhry SA. The Cholistan desert, A TOKTEN Consultancy Report. Bahawalpur: Cholistan Institute of Desert Studies, Islamia University; 1992. p. 34.

22. Ahmad F. Runoff farming in reducing rural poverty in Cholistan desert, Revista Sociedade \& Natureza, vol. 20. Brazil: Instituto de Geografia, Universidade Federal de Uberlândia; 2008. p. 177-88.

23. Punjab (Pakistan). Gazetteer of the Jhelum District, 1904, Part 1. Sang-e-Meel Publications; 1907

24. Rohtas Fort. [http://whc.unesco.org/en/list/586]

25. Taxila. [http://whc.unesco.org/en/list/139]

26. Encyclopaedia Britannica. [http://www.britannica.com/EBchecked/topic/ 472944/Potwar-Plateau]

27. Ali MA. Reconnaissance Soil Survey of the Rawalpindi Area. West Pakistan, Lahore: Directorate of Soil Survey; 1967. p. 129.

28. Ali N. Population and human settlement characteristics of Potohar region of Punjab (Pakistan), PhD Thesis. Peshawar: University of Peshawar; 2004.

29. ljaz SS, Ali S. Tillage and mulch effects on profile moisture dynamics fallow efficiency and rainfed wheat yields in Potowar. Pak J Agri Sci. 2007:44:90-5

30. Hussain M, Ahmed MSA, Hameed M, Aqeel M. Threats to rainfed and canal irrigated agro-ecosystems of the Punjab, Pakistan by weed infestation. Pak J Bot. 2012;44(SI):171-8

31. Martin GJ. Ethnobotany, A Method Manual. London: Chapman and Hall; 1995

32. Miles MB, Huberman AM. Qualitative Data Analysis: An Expanded Source Book. 2nd ed. London: Sage Publications; 1994.

33. Bridson D, Foreman L. The Herbarium Handbook. 3rd ed. Kew, Richmond, United Kingdom: Royal Botanic Gardens; 1998.

34. Silverman D. Interpreting qualitative data: methods for analyzing talk, text and interaction. 3rd ed. Thousand Oaks, California 91320: Sage Publications Inc; 1993.

35. Weber RP. Basic Content Analysis. 2nd ed. Newbury Park, California: Sage Publications; 1990.

36. Qureshi R, Waheed A, Arshad M, Umbreen T. Medico-ethnobotanical inventory of tehsil Chakwal, Pakistan. Pak J Bot. 2009;41:529-38.

37. Ahmed T, Kanwal R, Hassan M, Ayub N. Antibacterial activity of Solanum surrattense against water-borne Pathogens Isolated from Surface water of Pothwar region in Pakistan. Human Ecol Risk Assess. 2009;15:624-35.

38. Sonibare MA, Moody JO, Adesanya EO. Use of medicinal plants for the treatment of measles in Nigeria. J Ethnopharmacol. 2009;122:268-72.

39. Abbasi AM, Khan MA, Khan N, Shah MH. Ethnobotanical survey of medicinally important wild edible fruits species used by tribal communities of Lesser Himalayas-Pakistan. J Ethnopharmacol. 2013;148:528-36.

40. Ahmad M, Sultana S, Fazl-i-Hadi S, Ben Hadda T, Rashid S, Zafar M, et al. An Ethnobotanical study of Medicinal Plants in high mountainous region of Chail valley (District Swat-Pakistan). J Ethnobiol Ethnomed. 2014;10:36.

41. Murad W, Azizullah A, Adnan M, Tariq A, Khan KU, Waheed S, et al. Ethnobotanical assessment of plant resources of Banda Daud Shah, District Karak. Pak J Ethnobiol Ethnomed. 2013;9:77.
42. Ullah M, Khan MU, Mahmood A, Malik RN, Hussain M, Wazir SM, et al. An ethnobotanical survey of indigenous medicinal plants in Wana district south Waziristan agency, Pakistan. J Ethnopharmacol. 2013;150:918-24.

43. Mahmood A, Mahmood A, Malik RN. Indigenous knowledge of medicinal plants from Leepa valley, Azad Jammu and Kashmir, Pakistan. J Ethnopharmacol. 2012;143:338-46.

44. Rashid U, Khan MR, Jan S, Bokhari J, Shah NA. Assessment of phytochemicals, antimicrobial and cytotoxic activities of extract and fractions from Fagonia olivieri (Zygophyllaceae). BMC Complement Altern Med. 2013;13:167

45. Bibi Y, Nisa S, Chaudhary FM, Zia M. Antibacterial activity of some selected medicinal plants of Pakistan. BMC Complement Altern Med. 2011;11:52.

46. Hussain Z, Waheed A, Qureshi RA, Burdi DK, Verspohl EJ, Khan N, et al. The effect of medicinal plants of Islamabad and Murree region of Pakistan on insulin secretion from INS-1 cells. Phytother Res. 2004;18:73-7.

47. Malik F, Hussain S, Sadiq A, Parveen G, Wajid A, Shafat S, et al. Phyto-chemical analysis, anti-allergic and anti-inflammatory activity of Mentha arvensis in animals. Afr J Pharm Pharmacol. 2012;6:613-9.

48. Gulfraz M, Ahmad A, Asad MA, Sadiq A, Afzal U, Imran M, et al. Antidiabetic activities of leaves and root extracts of Justicia adhatoda Linn against alloxan induced high blood sugar in rats. Afr J Biotechnol. 2011;10:6101-6.

49. Bibi S, Sultana J, Sultana H, Malik RN. Ethnobotanical uses of medicinal plants in the highlands of Soan Valley, Salt Range, Pakistan. J Ethnopharmacol. 2014;155:352-61.

50. Shaheen H, Qureshi R, Akram A, Gulfraz M. Inventory of medicinal flora from Thal desert, Punjab, Pakistan. Afr J Tradit Compliment Altern Med. 2014;11:282-90.

51. Qureshi R, Maqsood M, Arshad M, Chaudhry AK. Ethnomedicinal uses of plants by the people of Kadhi areas of Khushab, Punjab, Pakistan. P J Bot. 2011:43:121-33.

52. Arshad M, Nisar MF, Majeed A, Ismail S, Ahmad M. Ethnomedicinal flora in district Sialkot, Punjab, Pakistan. Middle East J Sci Res. 2011;9:209-14.

53. Hameed M, Chaudhary AA, Main MA, Gill AH. Diversity of Plant Species in Lal Suhanra National Park, Bahawalpur, Pakistan. J Biol Sci. 2002;2:267-74.

54. Arshad M, Akbar G, Rashid S. Wealth of medicinal plants of Cholistan desert, Pakistan: conservational strategies. Hamdard Medicus. 2003;XLV:25-34.

55. Wariss HM. A Contribution to the Flora of Lal Suhanra National Park, Bahawalpur, M.Sc. Thesis. Multan: Govt. College Bosan Road Multan; 2006.

56. Hameed M, Ashraf M, Al-Quriany F, Nawaz T, Ahmad MSA, Younis A, et al. Medicinal Flora of the Cholistan Desert: A Review. Pak J Bot. 2011;43(Special Issue):39-50.

57. Shinwari, ZK. The Ethnobotany in Pakistan: Sustainable and Participatory Approach. Proc. 1st Training Workshop on Ethnobotany and its Application to Conservation NARC 1996, Islamabad, 14-25.

58. Wariss HM. Flora of the Cholistan Desert, M.Phil Thesis. Pakistan: The Islamia University of Bahawalpur; 2012.

59. Wariss HM, Mukhtar M, Anjum S, Bhatti GR, Pirzada SA, Alam K. Floristic composition of the plants of the Cholistan Desert, Pakistan. Am J Plant Sci. 2013;4:58-65.

60. Khadabadi SS, Bhajipale NS. A review on some important medicinal plants of Abutilon spp. Res J Pharmaceut Biol Chem Sci. 2010;1:718-29.

61. Jabeen A, Khan MA, Ahmad M, Zafar M, Ahmad F. Indigenous uses of economically important flora of Margallah Hills National Park, Islamabad, Pakistan. Afr J Biotechnol. 2009;8:763-84.

62. Qureshi R, Bhatti GR, Memon RA. Ethnomedicinal uses of herbs from northern part of Nara desert, Pakistan. Pak J Bot. 2010;42:839-51.

63. Marashdah MS, Al-Hazimi HM. Pharmacological activity of ethanolic extract of Alhagi maurorum roots. Arab J Chem. 2010:3:39-42.

64. Immanuel RR, Elizabeth LL. Weeds in agroecosystems: A source of medicines for human healthcare. Int J Pharm Tech Res. 2009;1:375-85.

65. Qureshi SJ, Khan MA. Ethnobotanical study of Kahuta from Rawalpindi District Pakistan. OnlineJ Biol Sci. 2001;1:27-30.

66. Arshad M, Ahmad M, Ahmed E, Saboor A, Abbas A, Sadiq S. An ethnobiological study in Kala Chitta hills of Pothwar region, Pakistan: multinomial logit specification. J Ethnobiol Ethnomed. 2014;10:13.

67. Sultana S, Khan MA, Ahmed M, Zafar M. Indigenous knowledge of folk herbal medicine by the women of district Chakwal Pakistan. Int Web J Ethnobotanical Leaflets. 2006:10:243-53.

68. Qureshi RA, Ahmed I, Ishtiaq M. Ethnobotanical and phytosociological studies of tehsil Gujar Khan district Rawalpindi. Asian J Plant Sci. 2006:5:890-3. 
69. Dalziel JM. Useful Plants of West Tropical Africa. London: Crown Agents for the Colonies; 1984

70. Katewa SS, Jain J. Aromatic and medicinal grasses of Aravalli hills of Rajasthan. In: Singh VK, Govil JN, Hashmi S, Singh G, editors. Ethnomedicine and Pharmacognosy II, vol. 7. USA: Sci Tech Publishers; 2003. p. 57.

71. Chopra RN, Nayar SL, Chopra IC. Glossary of Indian Medicinal Plants. New Delhi: National Institute of Science Communication and Information Resources; 2006. p. 80.

72. Rizk AM, El-Ghazaly GA. Medicinal and Poisonous Plants of Qatar. Doha: Modern Printing Press Ltd, University of Qatar, Scientific and Applied Research Centre; 1995.

73. Kataria S, Shrivastava B, Khajuria RK, Suri KA, Sharma P. Antimicrobial activity of Crotalaria burhia Buch.-Ham. roots. Ind J Nat Prod Resour. 2010;1:481-4.

74. Kumar RP, Rajesh K, Yogender M, Dharmesh S, Karthiyagini T. Standardization and preliminary phytochemical investigation on Cyperus rotundus Linn. rhizome. Int J Res Ayurveda Pharm. 2010;1:536-42.

75. Ahmed S, Ashraf M, Jabbar A, Janbaz KH. Biological activity of Dipterygium glaucum. Pak J Biol Sci. 2006;9:1173-4.

76. Natarajan D, Britto SJ, Srinivasan K, Nagamurugan N, Mohanasundari C, Perumal G. Anti-bacterial activity of Euphorbia fusiformis - a rare medicinal herb. J Ethnopharmacol. 2005;102:123-6.

77. Hayat MM, Sarwar S, Anjum S, Uzair M, Rasheed HMF, Jabeen Q, et al. Anti-diabetic and spasmolytic potential of Farsetia hamiltonii Royle from Cholistan desert. J Ethnopharmacol. 2014;156:347-52

78. Stella UY, Sasikala E, Rao GS, Sangeetha J. Pharmacognostic studies on Gisekia pharnacioides Linn. Ancient Sci Life. 2004:23:1-7.

79. Ahmad M, Eram S. Hepatoprotective studies on Haloxylon salicornicum: A plant from Cholistan desert. Pak J Pharm Sci. 2011:24:377-82.

80. Parvathamma S, Shanthamma C. Antimicrobial activity of Mollugo cerviana Ser. (Molluginaceae). Ancient Sci Life. 2000;20:1-4.

81. Rashid S, Iftekhar Q, Arshad M, lqbal J. Chemical composition and antibacterial activity of Suaeda fruticosa Forsk. from Cholistan, Pakistan. Pak J Biol Sci. 2000:3:348-9.

82. Ahmad M, Qureshi R, Arshad M, Khan M, Zafar M. Traditional herbal remedies used for the treatment of high blood sugar from district Attock (Pakistan). Pak J Bot. 2009;41:2777-82.

83. Abbasi AM, Khan MA, Ahmad M, Zafar M, Khan H, Muhammad N, et al. Medicinal plants used for the treatment of jaundice and liver diseases based on socio-economic documentation. Afr J Biotechnol. 2009:8:1643-50.

84. Hussain K, Zia-ul-Hussnain S, Shahazad A. Distributions and folk tibb knowledge of milk thistle (Silybum marianum L.) in NWFP, Pakistan. Ethnobot Leaflets. 2010;3:5.

\section{Submit your next manuscript to BioMed Central and take full advantage of:}

- Convenient online submission

- Thorough peer review

- No space constraints or color figure charges

- Immediate publication on acceptance

- Inclusion in PubMed, CAS, Scopus and Google Scholar

- Research which is freely available for redistribution 